\title{
Correlación entre la frecuencia de síntomas neuropsiquiátricos y de disfunción ejecutiva en pacientes con enfermedad de Alzheimer: Estudio exploratorio
}

\author{
Paloma A. Sotomayor, Patricio Fuentes G., \\ Andrea Slachevsky Ch.
}

El objetivo de este estudio es explorar correlaciones entre la frecuencia de síntomas neuropsiquiátricos y la disfunción ejecutiva en la enfermedad de Alzheimer (EA). Se trabajó con muestra de 39 pacientes con EA y se utilizó el NeuroPsychiatric Inventory Questionnarie (NPI-Q) para valorar la frecuencia de sintomas neuropsiquiátricos, el Frontal Assessment Battery (FAB) para observar la disfunción ejecutiva y el Mini Mental State Examination (MMSE) para dividir la muestra en subgrupos según deterioro cognitivo. Al culminar el estudio se encontraron correlaciones negativas entre la conducta de prehensión y la frecuencia de exaltación/euforia, trastornos del sueño, desinhibición y alucinaciones. Además se encontraron correlaciones positivas no esperadas entre sensibilidad a la interferencia y alucinaciones; secuencias motoras y desinhibición y trastornos de alimentación; fluencia verbal y trastornos de alimentación; y Go-No go y exaltación/euforia.

Enfermedad de Alzheimer / síntomas neuropsiquiátricos / disfunción ejecutiva / lóbulo frontal

Correlations between neuropsychiatric symptoms and executive dysfunction in Alzheimer's Disease

Objective: To explore the correlations between neuropsychiatric symptoms and executive dysfunction in Alzheimer's Disease (AD). Patients and method: In a sample of 39 patients with $A D$ the following instruments were used: the NeuroP sychiatric Inventory Questionnarie (NPI-Q) to assess the frequency of neuropsychiatric symptoms, the Frontal Assessment Battery (FAB) to assess executive dysfunction, and the Mini Mental State Examination (MMSE) to divide the sample into subgroups depending on level of cognitive impairment. Results: Negative correlations were found between prehension behavior and the frequency of euphoria/exaltation, sleep disorders, disinhibition and hallucinations.

Unexpected positive correlations were also found between sensitivity to interference and hallucinations; motor sequences and disinhibition and eating disorders; verbal fluency and eating disorders; and Go-No go and euphoria/exaltation.

Alzheimer's disease / neuropsychiatric symptoms / executive dysfunction / frontal lobe 
Las alteraciones neuropsiquiátricas del Alzheimer características de la enfermedad son apatía (72\%) y agitación $(60 \%)$, irritabilidad $(42 \%)$, ansiedad (48\%), disforia y conductas motoras anómalas (38\%), desinhibición (36\%), delusiones (22\%) y alucinaciones (10\%) (Cummings, 1997, 2003) independientemente del trasfondo cultural de la muestra de pacientes (Senanarong et al., 2005; Binetti et al., 1996; De Toledo et al., 2004). Piccininni et al. (2005) evaluaron la frecuencia y severidad de los síntomas neuropsiquiátricos en el Alzheimer a través del Inventario Neuropsiquiátrico NPI en 50 pacientes y reportan que los síntomas más frecuentes referidos por los cuidadores fueron apatía, conducta motora anómala, disforia y ansiedad (46-74\%).

Como manifestación de una disfunción del lóbulo frontal, Senanarong et al. (2004) observaron la prevalencia y características de la agitación en el Alzheimer y otras demencias, su asociación entre la agitación y otras variables clínicas y sus predictores. El estudio se realizó con 427 hombres y mujeres diagnosticados con demencias (277 con Alzheimer) evaluados con el Mini Mental State Examination (MMSE), Neuropsychiatric Inventory (NPI) y el Functional Activities Questionnaire (FAQ), tests neuropsicológicos y un instrumento de valoración del cuidador. Los autores encontraron en el grupo con EA una prevalencia aumentada de la agitación a medida que aumenta la severidad de la enfermedad. Los puntajes de las subescalas de desinhibición e irritabilidad se correlacionaron con la agitación a través de la severidad de la enfermedad. Los puntajes de las subescalas de conductas mediadas frontalmente, incluyendo la irritabilidad, delusiones y desinhibición predicen la mayor varianza en niveles de agitación en diferentes etapas de la enfermedad, aumentando con la severidad de la enfermedad. Concluyen que la agitación es común en la enfermedad de Alzheimer y otras demencias, con un marcado impacto sobre los cuidadores. Los datos sugieren que la agitación en el Alzheimer es un síndrome del lóbulo frontal. La disfunción del lóbulo frontal podría predisponer a los pacientes con Alzheimer a la agitación al exagerar las respuestas conductuales a varios tipos de psicopatologías coexistentes o provocaciones ambientales.

Senanarong et al. (2005), basándose en la hipótesis de que las actividades de la vida diaria dependen del planeamiento y memoria procedural, mediadas por los lóbulos frontales, investigaron la relación entre los síntomas neuropsiquiátricos, dibujo del reloj (planeamiento y juicio) y funciones ejecutivas en 73 pacientes diagnosticados con enfermedad de Alzheimer en Bangok, Tailandia. Se utilizaron como instrumentos de medición el Neuropsychiatric Inventory (NPI), la versión tailandesa del Mini Mental State Exa- 
mination (MMSE) como medida de la cognición global y la prueba del dibujo del reloj, la fluencia categorial (animales) y la fluencia fonológica (letras) como medidas ejecutivas. Para valorar las actividades de la vida diaria utilizaron la Escala de Actividades de la Vida Diaria Tailandesa, el Índice de Barthel y el Cuestionario de Valoración Funcional. Los resultados mostraron correlaciones estadísticamente significativas entre la copia del reloj (planeamiento y juicio) y las conductas frontalmente mediadas de agitación, apatía y desinhibición. La fluencia verbal se correlacionó con agitación, y los puntajes de la Escala de Actividades de la Vida Diaria se correlacionaron significativamente con los síntomas de agitación, apatía y desinhibición. Estos resultados sugieren una correlación entre conductas mediadas por el lóbulo frontal (funciones ejecutivas y síntomas neuropsiquiátricos) y un impedimento en las actividades de la vida diaria en ancianos tailandeses, que no se atribuye a un declive cognitivo general. Los autores concluyen que existe una correlación entre las actividades de la vida diaria y los cambios conductuales en ancianos tailandeses con enfermedad de Alzheimer. Afirman que el resultado enfatiza la relación importante entre los cambios conductuales característicos de la enfermedad y el deterioro en la funcionalidad de los pacientes, y el rol central que juega el deterioro del lóbulo frontal en el desarrollo de algunos de los aspectos que causan mayor incapacidad en la demencia.

En cuanto a la investigación de las funciones ejecutivas en la enfermedad de Alzheimer, Waltz et al. (2004) explican que al examinar los déficit de memoria en la EA con mayor detalle, existe amplia evidencia que sugiere que, en muchos casos, los déficit de memoria a corto plazo, en particular, pueden atribuirse a fallas del control ejecutivo (memoria de trabajo, por ejemplo) más que déficit en la memoria fonológica de corto plazo.

En un estudio acerca de la integración relacional y funciones ejecutivas en la enfermedad de Alzheimer, Waltz et al. (2004) clasificaron a pacientes con EA en subgrupos de acuerdo con su desempeño en funciones ejecutivas, utilizando medidas ejecutivas de memoria de trabajo y razonamiento que variaron sistemáticamente en cuanto a complejidad. Administraron medidas de memoria de trabajo (el Test de $\mathrm{N}$ atrás) y razonamiento (problemas de deducción de relaciones entre ítems) a pacientes con Alzheimer versus un grupo de control. Los pacientes mostraron déficit en las medidas de razonamiento que requerían una integración on-line de relaciones, pero se desempeñaron adecuadamente en ítems no relacionados e ítems que requerían el procesamiento de relaciones simples. Al subdividir el grupo de estudio, los pacientes que mostraron mayores dificultades en la tarea exhibieron un perfil neuropsi- 
cológico consistente con una disfunción prefrontal cortical.

Swanberg et al. (2004) señalan que estudios recientes sugieren que la disfunción ejecutiva es una manifestación común en la EA y ocurre en todas sus etapas. Citan varios estudios (Tekin et al., 2001), utilizando pruebas neuropsicológicas estándar de funciones ejecutivas, que han demostrado vínculos entre la disfunción ejecutiva y el declive funcional medido por escalas de actividades de la vida diaria.

Con el objetivo de determinar la prevalencia de la disfunción ejecutiva y estudiar su relación con síntomas cognitivos, funcionales y neuropsiquiátricos en la EA, Swanberg et al. (2004) realizaron un análisis retrospectivo de pacientes que participaron en el English Instruments Protocol of the Alzheimer's Disease Cooperative Study. Encontraron que un $64 \%$ de pacientes con EA fueron clasificados en la categoría de disfunción ejecutiva, con desempeños inferiores en tests de cognición, severidad de la demencia y actividades de la vida diaria y mayor frecuencia de síntomas de psicosis con mayor prominencia durante un periodo de 12 meses, en comparación con pacientes con funciones ejecutivas normales. Concluyen que los hallazgos apoyan la importancia de la valoración de las funciones ejecutivas en personas con EA y la importancia de la disfunción del lóbulo frontal en la EA.
Chen et al. (1998) hacen referencia a investigaciones que sugieren que la disfunción ejecutiva es común en la EA y se asocia a delusiones, progresión rápida de la demencia y una mayor necesidad de cuidados. En estudios de neuroimágenes se observa que los síntomas neuropsiquiátricos se asocian a disfunciones en diferentes regiones cerebrales específicas, y que la disfunción frontal es particularmente relevante a las expresiones no cognitivas de la enfermedad, por ejemplo los síntomas neuropsiquiátricos. Varios estudios reportan agitación, psicosis, depresión y apatía como disturbios neuropsiquiátricos comunes en la EA.

Dichos autores realizaron un estudio con el objetivo de relacionar las funciones ejecutivas, los síntomas neuropsiquiátricos y el estatus funcional en 31 pacientes diagnosticados con EA. Buscaron probar la hipótesis de que la disfunción ejecutiva se asocia a una mayor sintomatología neuropsiquiátrica y disfuncionalidad en la vida diaria.

Evaluaron siete aspectos de las funciones ejecutivas: fluencia verbal a través del Controlled Oral Word Association Test COWAT, conceptualización (similitudes, abstracción) e iniciación (secuencias motoras verbales, patrones) a través del Dementia Rating Scale DRS, categorización y perseveraciones (resolución de problemas) a través del Wisconsin Card Association Test WCST, y atención dividida y 
secuenciación a través del Trail Making Test B TMT-B.

Además, utilizaron el Mini Mental State Examination para valorar el estado cognitivo global, el Neuropsychiatric Inventory para evaluar la presencia y gravedad de síntomas neuropsiquiátricos y el Blessed Dementia Scale Activities Subscale para valorar las actividades de la vida diaria de los pacientes. Se halló una relación entre puntajes bajos en tests de funciones ejecutivas y mayor frecuencia de síntomas neuropsiquiátricos, una correlación más robusta entre la disfunción ejecutiva y la agitación/desinhibición, una correlación estadísticamente significativa entre bajos puntajes en funciones ejecutivas y factores de ansiedad y depresión, y una asociación significativa entre la disfunción ejecutiva asociada a la inhabilidad de llevar a cabo actividades de la vida diaria. Concluyen que la pérdida de las habilidades ejecutivas se acompaña de síntomas neuropsiquiátricos en la EA y es un posible marcador de esta, más allá de efectos de deterioro global cognitivo. La habilidad de llevar a cabo actividades de la vida diaria y cuidarse a uno mismo podría requerir de flexibilidad cognitiva y resistencia a la interferencia o distracción, independientemente del deterioro cognitivo global.

Johnson et al. (1999) realizaron un estudio con el objetivo de evaluar las características clínicas y patológicas de un subgrupo de pacientes con EA que exhibían déficit desproporcionadamen- te severos en tests de funcionamiento del lóbulo frontal, planteando un mayor grado de patología histológica en lóbulos frontales en comparación con la EA típica. Así, llevaron a cabo perfiles neuropsicológicos y estudios de placas seniles y ovillos neurofibrilares en las cortezas frontal, temporal, parietal y entorrinal en un grupo de 63 pacientes diagnosticados con EA.

En comparación con el grupo con EA típica, los pacientes en el grupo de estudio tuvieron desempeños significativamente inferiores en dos tests de funcionamiento frontal (Trail Making Test A y Fluencia verbal fonológica FAS) y en el test de Diseño de cubos de la escala de inteligencia para adultos de Weschler revisada. En el análisis de tejido cerebral encontraron cantidades comparables de ovillos neurofibrilares en todas las cortezas examinadas, a excepción de la corteza frontal, donde el grupo de estudio mostró mayor proporción que el grupo de EA típica.

Concluyen que identificaron un subgrupo de pacientes con patología de EA confirmada y en etapas iniciales de demencia, que presentaron déficit desproporcionadamente severos en test de funcionamiento del lóbulo frontal y con cantidades mayores de lo esperadas de marcadores patológicos en la corteza frontal, sugiriendo la existencia de una variante frontal en la enfermedad de Alzheimer. 


\section{Objetivos}

El objetivo general del estudio es observar las correlaciones entre síntomas neuropsiquiátricos y las funciones ejecutivas en una muestra de pacientes con enfermedad de Alzheimer.

Como objetivos específicos del trabajo se plantean:

- Describir la frecuencia de síntomas neuropsiquiátricos reportados por el cuidador, en una muestra chilena de pacientes diagnosticados con Alzheimer, a través de la aplicación del cuestionario neuropsiquiátrico Neuropsychiatric Inventory Questionnaire NPI-Q (Cummings et al., 1997).

- Describir el patrón de disfunción ejecutiva presentada por dichos pacientes, a través del análisis de los resultados de una batería neuropsicológica breve utilizada como screening de disfunciones ejecutivas, la Frontal Assessment Battery (FAB), (Dubois, 2000).

- Describir la frecuencia de síntomas neuropsiquiátricos a través del NPI$\mathrm{Q}$ en la muestra, creando tres subgrupos según la severidad del deterioro cognitivo (leve, moderado, severo), medido a través del Mini Mental State Examination MMSE, escala de valoración del deterioro cognitivo global creada por Folstein et al. en 1975.

- Explorar el patrón de disfunción ejecutiva característico de la mues- tra, a través de la FAB, haciendo la misma diferenciación por severidad del deterioro cognitivo.

- Establecer relaciones estadísticamente significativas entre síntomas neuropsiquiátricos y la disfunción ejecutiva en la enfermedad.

- Analizar si las relaciones encontradas entre los síntomas neuropsiquiátricos y las funciones ejecutivas en déficit difieren según la severidad del deterioro cognitivo de los pacientes.

\section{POBLACIÓN}

La muestra consiste en 39 pacientes diagnosticados con la enfermedad de Alzheimer, evaluados en el Hospital del Salvador en Santiago de Chile, durante los años 2004 y 2005.

A continuación se resume la información demográfica de la muestra (tabla 1).

Tabla 1

Promedios (desviación estándar) del grupo en cuanto a características demográficas

\begin{tabular}{ll}
\hline & Media (DS) \\
\hline Género (M/F) & $13 / 26$ \\
Edad & $76.6(7.1)$ \\
Años de estudio & $8.2(5.6)$ \\
\hline
\end{tabular}

La muestra se compone de 13 hombres y 26 mujeres, con una edad promedio de 76.6 (7.1) años y un promedio de años de estudio de 8.2 (5.6). Las edades van desde los 56 hasta los 85 años (gráfico 1). 


\section{Gráfico 1}

Distribución de la muestra por edad

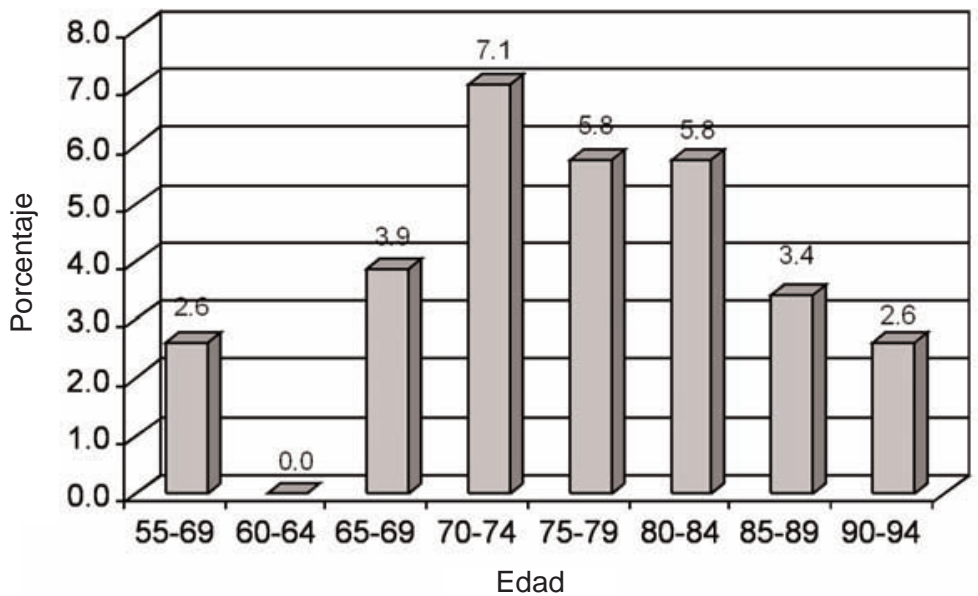

La muestra esta compuesta por 39 sujetos con edades que van de los 56 años a los 93 años de edad. Las edades más frecuentes en la muestra son 74 años, 77 años y 80 años. El grupo de edad más frecuente es de 70 a 74 años.

\section{Metodología}

La presente investigación correlacional es de tipo exploratoria, con un diseño de divisiones cruzadas, realizada con el propósito de destacar los aspectos fundamentales de la problemática planteada como tema de investigación, y encontrar los procedimientos adecuados para elaborar una investigación posterior. Es importante considerar para el análisis de los resultados de la presente investigación, que la ejecución esperada para pacientes que no mostraran una patología es de un mayor puntaje en las pruebas de eficiencia cognitiva global
(MMSE) y funciones ejecutivas (FAB), $y$ de un menor puntaje en la frecuencia de síntomas neuropsiquiátricos (NPI-Q).

\begin{tabular}{ll}
\hline Prueba & Ejecución normal \\
\hline MMSE & 30 puntos \\
FAB & 18 puntos \\
NPI-Q & 0 puntos \\
\hline
\end{tabular}

INSTRUMENTOS DE INVESTIGACIÓN

Mini Mental State Examination MMSE (Folstein, Folstein y McHugh en 1975)

Es un test de screening de eficiencia cognitiva global que consiste en 11 ítems que exploran funciones cognitivas principales, requiriendo de respuestas verbales para la evaluación de la orientación temporal y espacial, atención y cálculo, memoria a corto plazo, lenguaje (incluyendo repetición, 
nominación, comprensión de órdenes simples y complejas, y lectoescritura); y respuestas motoras para la exploración de las habilidades constructivas y praxias. El test tiene un puntaje máximo de 30 puntos. En estudios poblacionales se ha establecido un punto de corte para demencias de 24 puntos (Mc Dowell \& Newel, 1996) (anexo 1).

\section{Neuropsychiatric Inventory Questionnaire NPI-Q (Kaufer et al., 2000)}

Es un test de screening de los síntomas neuropsiquiátricos más comunes de las demencias, adaptado del Neuropsychiatric Inventory de Cummings et al. (1994). Consiste en una encuesta autoaplicada retrospectiva (1 mes) al cuidador acerca de la severidad y estrés que causa en el cuidador abarcando 12 dominios neuropsiquiátricos, incluye delirios, alucinaciones, trastornos del estado de ánimo, sueño y alimentación y conductas motoras anómalas (anexo 2).

Para el presente estudio solo se tomó en cuenta el puntaje de frecuencia de aparición de los síntomas, y no su gravedad ni el estrés que causa en el cuidador.

\section{Frontal Assessment Battery FAB (Dubois et al., 2000)}

Es un test de screening de funciones ejecutivas, que consiste en seis componentes de funciones ejecutivas: Conceptualización y razonamiento (analo- gías), Flexibilidad mental (fluencia verbal), Programación motora y control ejecutivo de la acción (secuencias motoras), Resistencia a la interferencia (instrucciones conflictivas), Control inhibitorio (Go-No go), Autonomía del ambiente (Prehensión), (anexo 3).

La correcta ejecución de cada subprueba recibe un puntaje de 3 , llevando a un puntaje máximo de 18 para la prueba. Rodríguez del Álamo et al. (2003) localizan el punto de corte para el déficit frontosubcortical en 16-15, y el punto de corte para la demencia frontosubcortical en 13-12.

\section{Procedimiento de ReCOlección de DATOS}

La recolección de datos se llevó a cabo en el Hospital del Salvador, en Santiago de Chile, durante los años 2004 y 2005. Todos los pacientes fueron evaluados por dos psicólogos licenciados, con un tiempo de entrenamiento de un año y medio en el uso de instrumentos neuropsicológicos y evaluación en demencias, dos pasantes en la Unidad de Neurología Cognitiva y Demencias del Hospital del Salvador y estudiantes del Diplomado de Neuropsicología de la Universidad Católica de Chile, bajo supervisión de los primeros.

Los sujetos que componen la muestra fueron pacientes de la Unidad de Neurología Cognitiva y Demencias de dicho hospital. Tras la consulta con neurólogos, cada paciente fue evaluado 
en un lapso de dos horas por sesión individual, hasta dos sesiones, por dos psicólogos y dos pasantes, encargados de aplicar una batería neuropsicológica completa, en la cual se aplicaron las pruebas consideradas para este estudio.

Se aplicó una batería neuropsicológica completa a cada uno de los pacientes, así como cuestionarios de comportamiento y funcionalidad a los familiares o acompañantes.

La Evaluación Neuropsicológica constituye un protocolo de atención aplicado sistemáticamente a pacientes con demencia en la Unidad de Neurología Cognitiva del Hospital del Salvador.

\section{Resultados}

\section{Análisis descriptivo}

Los sujetos que componen la muestra alcanzan un puntaje promedio en el Mini Mental State Examination de 17.7, puntaje correspondiente a un deterioro cognitivo moderado. En el Frontal Assessment Battery, la muestra total alcanza un puntaje promedio de 8.5 , el cual corresponde a una disfunción ejecutiva severa. Respecto a los síntomas neuropsiquiátricos, la puntuación media señala una frecuencia de 5.3 síntomas neuropsiquiátricos en la muestra (tabla 2).

Al separar la muestra en subgrupos, según deterioro cognitivo, encontramos:

Tabla 2

Medias y desviación estándar de los puntajes alcanzados por la muestra en las pruebas aplicadas

\begin{tabular}{|c|c|c|c|c|c|c|c|c|c|c|c|}
\hline \multirow[b]{2}{*}{$\begin{array}{l}\text { Deterioro } \\
\text { congnitivo }\end{array}$} & \multirow[b]{2}{*}{$\mathrm{n}$} & \multirow[b]{2}{*}{ MMSE } & \multirow[b]{2}{*}{$\begin{array}{l}\text { FAB } \\
\text { Total }\end{array}$} & \multicolumn{6}{|c|}{ FAB ítems } & \multirow{2}{*}{$\begin{array}{l}\text { NPI-Q } \\
\text { total }\end{array}$} & \multirow{2}{*}{$\begin{array}{l}\text { NPI-Q Síntomas } \\
\text { más frecuentes }\end{array}$} \\
\hline & & & & $\begin{array}{c}\text { Conceptua- } \\
\text { lización }\end{array}$ & $\begin{array}{c}\text { Fluidez } \\
\text { verbal }\end{array}$ & $\begin{array}{c}\text { Secuencias } \\
\text { motoras }\end{array}$ & $\begin{array}{l}\text { Sensibilidad } \\
\text { interferencial }\end{array}$ & $\begin{array}{l}\text { Go- } \\
\text { No go }\end{array}$ & $\begin{array}{c}\text { Prehen- } \\
\text { sión }\end{array}$ & & \\
\hline Muy leve & 5 & $\begin{array}{c}28.8 \\
81.309\end{array}$ & $\begin{array}{c}10.4 \\
(3.65)\end{array}$ & $\begin{array}{c}0.6 \\
(1.349\end{array}$ & $\begin{array}{c}1.6 \\
(0.89)\end{array}$ & $\begin{array}{c}2 \\
(1.00)\end{array}$ & $\begin{array}{c}2.6 \\
(0.89)\end{array}$ & $\begin{array}{c}1.8 \\
(1.30)\end{array}$ & $\begin{array}{c}1.8 \\
(1.10)\end{array}$ & $\begin{array}{c}5.2 \\
(2.77)\end{array}$ & $\begin{array}{l}\text { Agitación/agresión, } \\
\text { ansiedad, apatía/ } \\
\text { indiferencia, } \\
\text { desinhibición, irrita- } \\
\text { bilidad/labilidad }\end{array}$ \\
\hline Leve & 13 & $\begin{array}{c}21.4 \\
(1.71)\end{array}$ & $\begin{array}{c}11.6 \\
(3.73)\end{array}$ & $\begin{array}{c}1.7 \\
81.03)\end{array}$ & $\begin{array}{c}2.1 \\
(0.86)\end{array}$ & $\begin{array}{c}1.4 \\
(0.96)\end{array}$ & $\begin{array}{c}2.2 \\
(1.28)\end{array}$ & $\begin{array}{c}1.6 \\
(1.199\end{array}$ & $\begin{array}{c}2.7 \\
(0.75)\end{array}$ & $\begin{array}{c}4.9 \\
(3.53)\end{array}$ & $\begin{array}{c}\text { Depresión/disforia, } \\
\text { ansiedad }\end{array}$ \\
\hline Moderado & 15 & $\begin{array}{c}15.6 \\
(1.72)\end{array}$ & $\begin{array}{c}7.8 \\
(4.13)\end{array}$ & $\begin{array}{c}0.7 \\
(0.90)\end{array}$ & $\begin{array}{c}1.2 \\
(0.77)\end{array}$ & $\begin{array}{c}1.1 \\
(0.52)\end{array}$ & $\begin{array}{c}1.7 \\
(1.23)\end{array}$ & $\begin{array}{c}1.1 \\
(1.339\end{array}$ & $\begin{array}{c}2.1 \\
(1.22)\end{array}$ & $\begin{array}{c}4.9 \\
(2.88)\end{array}$ & $\begin{array}{c}\text { Ansiedad, } \\
\text { apatía/indiferencia }\end{array}$ \\
\hline Severo & 6 & $\begin{array}{c}8.3 \\
(2.42)\end{array}$ & $\begin{array}{c}2.0 \\
(1.10)\end{array}$ & $\begin{array}{c}0.3 \\
(0.52)\end{array}$ & $\begin{array}{c}0.0 \\
(0.00)\end{array}$ & $\begin{array}{c}0.2 \\
(0.41)\end{array}$ & $\begin{array}{c}0.0 \\
(0.00)\end{array}$ & $\begin{array}{c}0.2 \\
(0.41)\end{array}$ & $\begin{array}{c}1.3 \\
(1.37)\end{array}$ & $\begin{array}{c}7.2 \\
(3.43)\end{array}$ & $\begin{array}{l}\text { Depresión/disforia, } \\
\text { conducta motora } \\
\text { anómala, trastornos } \\
\text { del sueño }\end{array}$ \\
\hline $\begin{array}{l}\text { Muestra } \\
\text { total }\end{array}$ & 39 & $\begin{array}{c}17.7 \\
(5.64)\end{array}$ & $\begin{array}{c}8.5 \\
(4.78)\end{array}$ & $\begin{array}{c}0.9 \\
(1.07)\end{array}$ & $\begin{array}{c}1.4 \\
(1.01)\end{array}$ & $\begin{array}{c}1.2 \\
(0.88)\end{array}$ & $\begin{array}{c}1.7 \\
(1.349\end{array}$ & $\begin{array}{c}1.2 \\
(1.26)\end{array}$ & $\begin{array}{c}2.1 \\
(1.15)\end{array}$ & $\begin{array}{c}5.3 \\
(3.15)\end{array}$ & $\begin{array}{c}\text { Ansiedad, } \\
\text { apatía/indiferencia }\end{array}$ \\
\hline
\end{tabular}


- El subgrupo de deterioro cognitivo muy leve alcanza un puntaje promedio en el MMSE de 25.8, FAB de 10.4 y una mayor frecuencia de agitación/agresión, ansiedad, apatía /indiferencia, desinhibición e irritabilidad/labilidad.

- El subgrupo de deterioro cognitivo leve alcanza un puntaje promedio en el MMSE de 21.4, FAB de 11.6 y presenta una mayor frecuencia de depresion/disforia y ansiedad.

- El subgrupo de deterioro cognitivo moderado alcanza un puntaje promedio en el MMSE de 15.6, FAB de 7.8 y presenta una mayor frecuencia de ansiedad y apatía/indiferencia.

- El subgrupo de deterioro cognitivo severo alcanza un puntaje promedio en el MMSE de 8.3, FAB de 2.0 y presenta una mayor frecuencia de depresion/disforia, conductas motoras anómalas y trastornos del sueño.
El ítem con menor puntaje en la muestra es el de Conceptualización, indicando un mayor deterioro de la capacidad de abstracción entre los sujetos de la muestra.

Por otro lado, el ítem de Prehensión alcanza la mayor puntuación media, indicando una preservación del reflejo de prehensión en la muestra.

\section{Desempeño de la muestra por pruebas}

Respecto al nivel de deterioro cognitivo, medido por el Mini Mental State Examination, el $12,8 \%$ de la muestra recibe un puntaje indicativo de un Deterioro cognitivo muy leve; el 33,3\% recibe un puntaje correspondiente a un Deterioro cognitivo leve; el 38,5\% de la muestra recibe puntajes que pertenecen a la categoría de Deterioro cognitivo moderado; y el 15,4\% logran puntajes que corresponden a la categoría de Deterioro cognitivo severo (gráfico 2).

\section{Gráfico 2}

Distribución de la muestra por severidad de deterioro cognitivo según puntaje obtenido en el Mini Mental State Examination

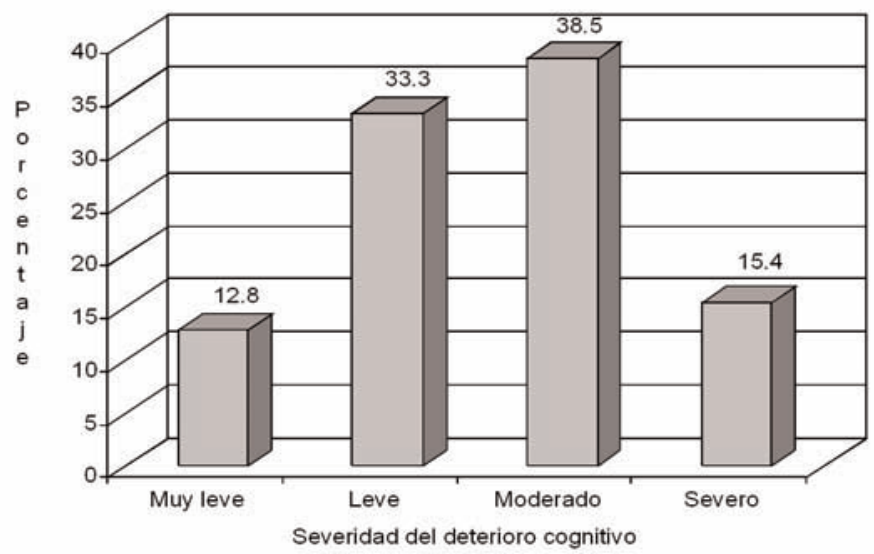


Gráfico 3

Distribución de la muestra por puntaje obtenido en el Frontal Assessment Battery

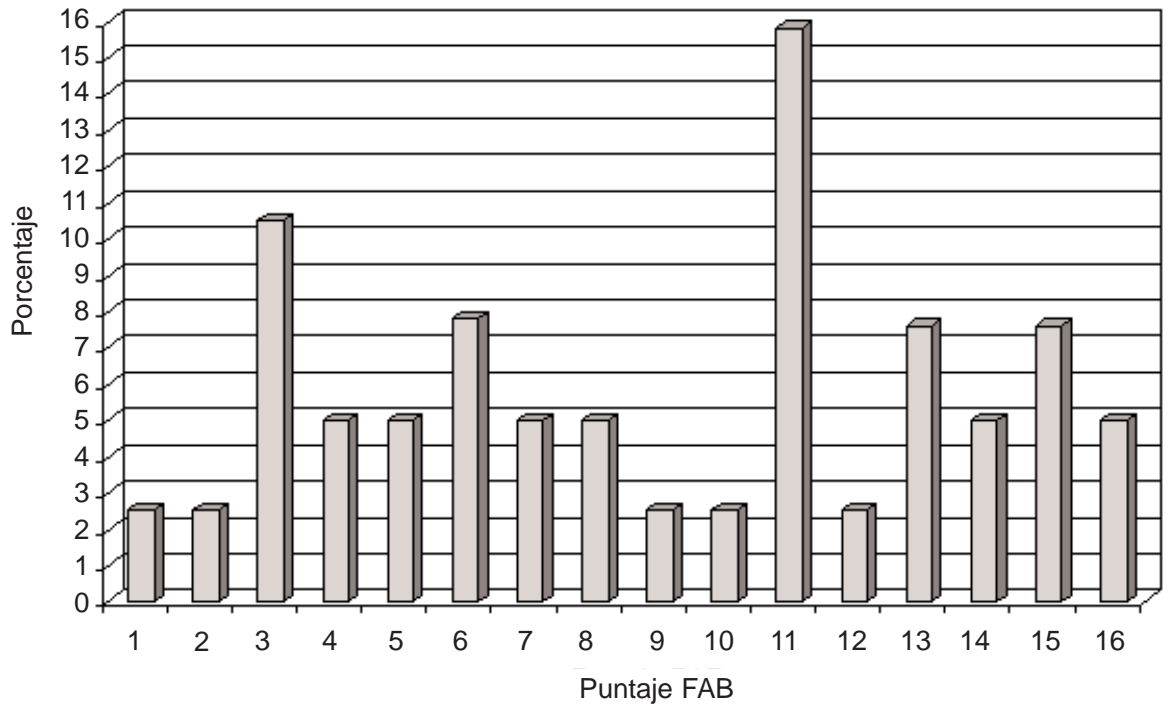

Por otro lado, se observó la muestra según los puntajes obtenidos en la batería de evaluación de disfunción ejecutiva Frontal Assesment Battery (FAB). El 82,2\% de la muestra alcanza puntajes inferiores a 13 puntos, lo cual corresponde al desempeño mostrado en estudios por pacientes diagnosticados de Demencia frontosubcortical $(\mathrm{Du}-$ bois et al., 2000). En otras palabras, muestran un desempeño alterado en pruebas de función ejecutiva. El 17,9\% restante muestra un déficit frontosubcortical, con puntajes inferiores a 16 , lo cual se traduce en una disminución en las capacidades ejecutivas. Ningún paciente alcanza puntajes de 17-18, los cuales se consideran normales, es decir, sin déficit de las funciones ejecutivas (gráfico 3).

$\mathrm{Al}$ analizar la ejecución de la muestra en la FAB por ítem, observamos que el $48,7 \%$ de los sujetos no reciben puntaje para conceptualización, indicando dificultades para abstraer similaridades entre dos conceptos pertenecientes a una misma categoría semántica. El $35,9 \%$ de los sujetos reciben puntaje de 2 en el ítem de fluidez, indicando dificultades para generar información a partir de letra A (flexibilidad mental espontánea), y solo logran generar menos de 10 palabras. El 66,6\% de los sujetos recibe un puntaje de 1 en el ítem de programación motora, indicando dificultades de programación de una secuencia motora. El 43,6\% de los sujetos reciben un puntaje completo (3) en el ítem de sensibilidad a la interferencia, indicando que la capacidad de lle- 
var a cabo instrucciones conflictivas se encuentra preservada. El 43,6\% de los sujetos no recibe puntaje en el ítem de Go-No Go, indicando dificultades para inhibir respuestas automáticas (control de impulsos). El 61,5\% de los sujetos reciben un puntaje de 3 en el ítem de Conducta y Prehensión, lo cual señala que no presentan el reflejo de prehensión (signo de liberación frontal) (gráfico 4).

Al observar el desempeño de la muestra en el Cuestionario de Síntomas Neuropsiquiátricos se observa que el 53,8\% de los sujetos pertenecientes a

la muestra presentan hasta cinco síntomas neuropsiquiátricos $(\leq 5)$, mientras que el $43,7 \%$ muestra hasta 11 síntomas $(\leq 11)$ (gráfico 5).

En el análisis del desempeño de la muestra en los ítems del cuestionario neuropsiquiátrico encontramos (gráfico 6) que los síntomas neuropsiquiátricos que se presentan con mayor frecuencia entre los sujetos de la muestra son ansiedad (61,5\%), depresión $(56,4 \%)$ y apatía y/o indiferencia $(56,4 \%)$. Los síntomas que se presentan con menor frecuencia son exaltación y/o euforia $(5,1 \%)$, alucinaciones $(28,2 \%)$ y desinhibición $(33,3 \%)$.

\section{Gráfico 4}

Desempeño de la muestra en la FAB: Análisis del puntaje obtenido por ítem Puntaje $\square \square 0 \quad \square 1 \quad \square 2 \quad \square 3$

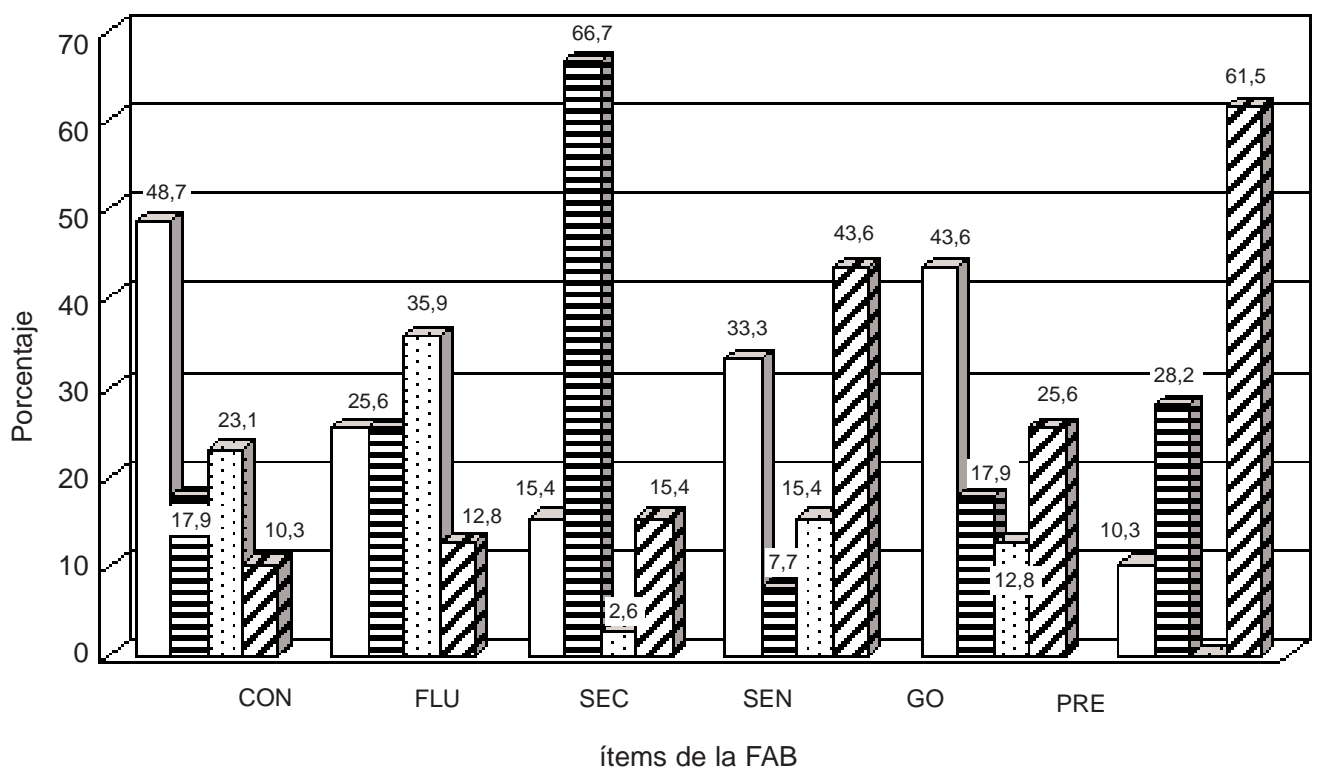

CON = Conceptualización, FLU = Fluencia verbal, SEC = Secuencias motoras, GO = Go-No go (control inhibitorio), PRE = Prehensión (adherencia al medio). 


\section{Gráfico 5}

Distribución de la muestra por frecuencia de síntomas neuropsiquiátricos según el Neuro Psychiatric Inventory Questionnaire NPI-Q

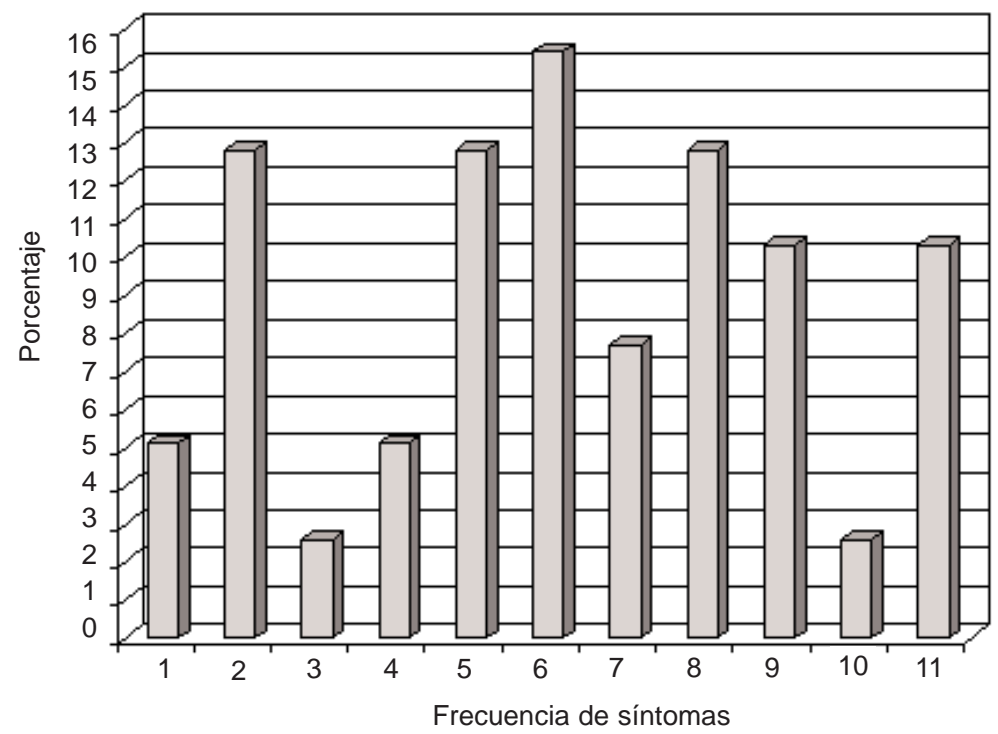

Gráfico 6

Frecuencia de síntomas en el NPI-Q

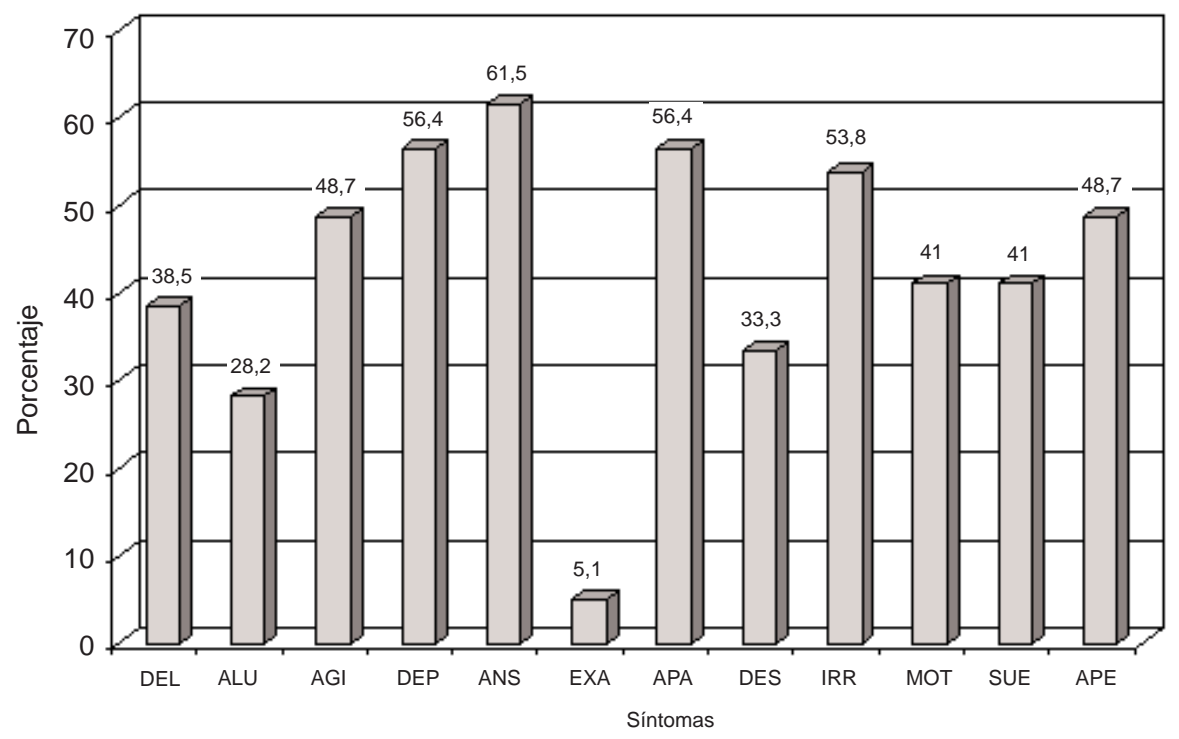

$\mathrm{DEL}=$ Delusiones, $\mathrm{ALU}=$ Alucinaciones, $\mathrm{AGI}=$ Agitación/agresividad, $\mathrm{DEP}=$ Depresión $/$ disforia, ANS $=$ Ansiedad, EXA = Exaltación/euforia, APA = Apatía/indiferencia, $\mathrm{DES}=$ Desinhibición, $\mathrm{IRR}=$ Irritabilidad/labilidad, MOT = Conductas motoras anómalas, SUE = Trastornos del sueño, $\mathrm{APE}=$ Trastornos del apetito/alimentación. 


\section{Correlaciones no paramétricas}

Las correlaciones encontradas en el presente estudio fueron (tabla 3):

- Muestra total: Se encontraron correlaciones negativas entre la conducta de prehensión y la frecuencia de exaltación/euforia y trastornos del sueño. A menor puntaje en el ítem de conducta de prehensión (indicativo de patología), mayor frecuencia de exaltación/euforia y trastornos del sueño en la muestra total.

- Grupo deterioro cognitivo muy leve: Correlación perfecta entre sensibilidad a la interferencia y la frecuencia de alucinaciones. A mayor puntaje en el ítem de sensibilidad a la interferencia (indicativo de preservación de resistencia ante la interferencia), mayor frecuencia de alucinaciones en el grupo.

- Grupo deterioro cognitivo leve: Se encontraron correlaciones negativas entre la conducta de prehensión y la frecuencia de exaltación/euforia, conducta de prehensión y frecuencia de desinhibición y una correlación positiva entre secuencias motoras y frecuencia de desinhibición, y secuencias motoras y trastornos de alimentación. A menor puntaje en el ítem de conducta de prehensión (indicativo de patología), es mayor la frecuencia de exalta- ción/euforia y desinhibición. Por otro lado, a mayor puntaje en el ítem de secuencias motoras (indicativo de preservación de la capacidad de programación motora), mayor frecuencia de desinhibición y trastornos de la alimentación.

- Grupo deterioro cognitivo modera$d o$ : Se encontraron correlaciones negativas entre la conducta de prehensión y la frecuencia de trastornos del sueño, conducta de prehensión y frecuencia de alucinaciones; y una correlación positiva entre la fluencia verbal y la frecuencia de trastornos de alimentación. A menor puntaje en el ítem de conducta de prehensión (indicativo de patología), mayor la frecuencia de trastornos del sueño y alucinaciones. Además, a mayor puntaje en el ítem de fluencia verbal (indicativo de preservación de la capacidad de generación de información), mayor frecuencia de trastornos de la alimentación.

- Grupo deterioro cognitivo severo: Se encontró una correlación perfecta entre el ítem Go-No go y la frecuencia de exaltación/euforia. A mayor puntaje en el ítem de Go-No go (indicativo de preservación de la capacidad de control de impulsos), mayor frecuencia de conductas de exaltación/euforia en el grupo. 
Tabla 3

Cuadro resumen de correlaciones encontradas

\begin{tabular}{|l|l|c|c|c|c|c|}
\hline & & \multicolumn{3}{|c|}{ Ítems } \\
\hline $\begin{array}{l}\text { Severidad del } \\
\text { deterioro cognitivo }\end{array}$ & \multicolumn{1}{|c|}{ Ítems FAB } & $\begin{array}{l}\text { Exaltación/ } \\
\text { euforia }\end{array}$ & Sueño & Alucinaciones & Desinhibición & Alimentación \\
\hline Muestra total & $\begin{array}{l}\text { Conducta de } \\
\text { prehensión }\end{array}$ & $-0.338^{*}$ & $-0.44^{* *}$ & - & - & - \\
\hline \multirow{2}{*}{ Muy leve } & $\begin{array}{l}\text { Sensibilidad a la } \\
\text { interferencia }\end{array}$ & - & - & $1.000^{* *}$ & - & - \\
\hline \multirow{2}{*}{ Meve } & $\begin{array}{l}\text { Conducta de } \\
\text { prehensión }\end{array}$ & $-0.674^{* *}$ & - & - & $-0.632^{*}$ & - \\
\cline { 2 - 7 } & $\begin{array}{l}\text { Secuencias motoras } \\
\text { Moderado }\end{array}$ & - & - & - & $0.769^{* *}$ & $0.609^{*}$ \\
\cline { 2 - 7 } & prehensión & - & $-0.678^{* *}$ & $-0.519^{*}$ & - & - \\
\hline Severo & Gluencia verbal & - & - & - & - & $0.797^{* *}$ \\
\hline
\end{tabular}

* La correlación es significativa al nivel 0,05 (bilateral)

** La correlación es significativa al nivel 0,01 (bilateral)

\section{DISCUSIÓN}

La mayor parte de la muestra alcanza desempeños correspondientes a un deterioro cognitivo moderado (MMSE 17.7), con un puntaje promedio en la FAB de 8.5 puntos, indicativo de un déficit frontal córtico-subcortical grave, y un promedio de hasta cinco síntomas neuropsiquiátricos.

En la evaluación de la disfunción ejecutiva, el ítem que mayor dificultad presentó para los sujetos de la muestra fue Conceptualización, indicando problemas para abstraer similaridades entre dos conceptos pertenecientes a una misma categoría semántica. La mayor parte de la muestra no alcanzó ningún puntaje en este ítem. Se observó que, con frecuencia, las respuestas dadas a este ítem consistían en similitudes concretas entre los objetos presentados, especialmente frente a los objetos mesasilla, donde se observó la tendencia a responder que se parecen en que son para sentarse o son de madera. Dubois et al. (2000) reportan este tipo de respuestas como características de pacientes con disfunción frontal, dada una dificultad de razonamiento abstracto.

El ítem Go-No go representó el segundo ítem más difícil para la muestra, indicando dificultades para el control de impulsos. Se observó que la mayor parte de pacientes pudo ejecutar la imitación (responder con un golpe cuando el evaluador ejecuta un golpe), pero encontraron difícil inhibir la tendencia a imitar los dos golpes del evaluador, 
aunque durante la práctica sí lo pudieran ejecutar adecuadamente (frente a dos golpes del evaluador, el paciente no golpea). Este patrón de respuesta ha sido descrito en pacientes con lesiones frontales, en especial en el área ventral de los lóbulos frontales (Slachevsky et al., 2005).

Por otro lado, en el ítem de Programación motora la mayor parte de la muestra alcanza un puntaje de 1 , indicando una dificultad para la secuenciación de una serie de tres movimientos con la mano. Este resultado indica que la mayor parte de la muestra sí fue capaz de imitar la secuencia motora, realizándola junto con el evaluador, pero encontraron difícil ejecutar seis movimientos consecutivos de la secuencia por sí mismos. Se observa una dificultad en aprender el orden de los gestos que componen la muestra y una tendencia a alterar alguno de los gestos. Lipton et al. (2005) describen que el ítem de la FAB que mejor discrimina entre pacientes diagnosticados con enfermedad de Alzheimer y pacientes con demencia frontotemporal es el de Programación motora; el grupo de pacientes con EA tiene desempeños inferiores que el de los pacientes con demencia frontotemporal. Neuroanatómicamente podríamos explicar esta dificultad como consecuencia de la degeneración hipocampal (dificultad de aprendizaje), del área de prefrontales (déficit en la programación de secuencias motoras per se) o de áreas parietales posteriores (apraxias). Establecer esta diferenciación sería motivo de mayores estudios.

En el ítem de fluidez verbal, la mayor parte de la muestra alcanza un puntaje de 2, indicando que pudieron generar entre seis y 10 palabras con la letra A en un minuto. Este resultado refleja una disminución de la fluencia verbal fonológica (flexibilidad mental), dato que se ha reportado en investigaciones como una disminución en la capacidad de generación de palabras (Cummings et al., 1988; Baudic et al., 2006).

La mayor parte de la muestra no encontró dificultades en el ítem de Conducta de prehensión, lo cual indica la ausencia del reflejo primitivo de prehensión (tomar las manos del evaluador). Este reflejo es altamente indicativo de una disfunción frontal, y se observa con más frecuencia en las demencias de tipo frontal (Lhermitte et al., 1986) o vascular (Hogan, 1995).

El ítem de sensibilidad a la interferencia también se encontró preservado en la mayor parte de la muestra, indicando la ausencia de dificultades para seguir instrucciones conflictivas.

Observamos que los síntomas neuropsiquiátricos de agitación, ansiedad, apatía y depresión descritos comúnmente en la literatura como parte del perfil neuropsiquiátrico de la enfermedad de Alzheimer (Cummings, 1997, 2003), aparecen en la muestra desde etapas tempranas de deterioro cognitivo. La ansiedad se muestra consistente- 
mente a lo largo de la muestra, independientemente del nivel de deterioro cognitivo alcanzado.

Por otro lado, las conductas motoras anómalas (vagabundeo) y los trastornos del sueño, que fueron más frecuentes en el subgrupo de deterioro cognitivo severo, se han descrito en la enfermedad de Alzheimer desde etapas moderadas de la enfermedad (Mesulam, 2000). Los síntomas neuropsiquiátricos que se presentaron con más frecuencia en la muestra fueron ansiedad $(61,5 \%)$, apatía/indiferencia $(56,4 \%)$, depresión/disforia $(56,4 \%)$, irritabilidad/labilidad $(53,8 \%) \quad y \quad$ agitación/agresividad $(48,7 \%)$. La frecuencia de estos síntomas han sido ampliamente descritos en la enfermedad de Alzheimer (Cummings, 1997, 2003; Chen et al., 1998; Piccininni et al., 2005; Rosenberg et al., 2005; Benoit et al., 2005; Senanarong, 2005). Los síntomas neuropsiquiátricos menos frecuentes en la muestra fueron la exaltación/euforia, alucinaciones y desinhibición, síntomas que han sido descritos como más frecuentes en otros tipos de demencias, por ejemplo las demencias frontales, cuya presencia en la enfermedad de Alzheimer es más tardía (Craig et al., 2005).

En la muestra total se encontraron correlaciones significativas negativas entre la conducta de prehensión y la frecuencia de exaltación/euforia y trastornos del sueño. A menor puntaje en el ítem de conducta de prehensión (indi- cativo de patología), mayor frecuencia de exaltación/euforia y trastornos del sueño. La conducta de prehensión es considerada uno de los reflejos primitivos característicos de pacientes con lesiones frontales. $\mathrm{Su}$ presencia es altamente indicativa de una patología y es considerada una de las conductas de adherencia descritas por Lhermitte et al. (1986).

Por otro lado, las conductas de exaltación y euforia se consideran como parte de un cuadro de hiperactividad global, mostrado por pacientes con lesiones frontales, especialmente en la corteza orbitofrontal. Está descrito en la literatura de los trastornos de la corteza frontal la aparición de ambos síntomas como parte de los trastornos del comportamiento característicos de la patología frontal (Slachevsky et al., 2005).

Las relaciones entre la disfunción ejecutiva y la frecuencia de síntomas neuropsiquiátricos se dan a lo largo de todos los subgrupos, sugiriendo que posiblemente esta relación se presente independientemente del deterioro cognitivo global. Chen et al. (1998) reportan haber encontrado relaciones significativas entre la disfunción ejecutiva y los síntomas neuropsiquiátricos independientes de los puntajes del MMSE, interpretando dicho resultado como la posibilidad de una mayor especificidad para déficit ejecutivos, que se extiende más allá de las habilidades cognitivas globales. 
En el grupo de deterioro cognitivo muy leve se observa una correlación significativa perfecta entre sensibilidad a la interferencia y la frecuencia de alucinaciones. A mayor puntaje en el ítem de sensibilidad a la interferencia (indicativo de preservación de resistencia ante la interferencia), mayor frecuencia de alucinaciones en el grupo que sufre de un deterioro cognitivo muy leve. Este es un resultado no esperado y consiste en una interrogante interesante para estudios posteriores.

En el grupo de deterioro cognitivo leve se encontraron correlaciones significativas negativas entre la conducta de prehensión y la frecuencia de exaltación/euforia y entre la conducta de prehensión y la frecuencia de desinhibición, y una correlación significativa positiva entre secuencias motoras y frecuencia de desinhibición y secuencias motoras y trastornos de alimentación. A menor puntaje en el ítem de conducta de prehensión, indicativo de patología, mayor la frecuencia de exaltación/euforia y desinhibición. Este resultado está acorde con lo reportado para la patología frontal descrita comúnmente en la literatura. Pacientes que manifiestan trastornos del comportamiento secundarios a lesión o disfunción frontal, usualmente muestran el reflejo de prehensión y otras conductas de adherencia al medio, además de conductas hiperactivas, como desinhibición y exaltación (Slachevsky et al., 2005). Por otro lado, se observa en este grupo que a mayor puntaje en el ítem de secuencias motoras, indicativo de la preservación de la capacidad de programación motora, mayor frecuencia de desinhibición y trastornos de la alimentación, resultado que no era esperado en la presente investigación.

En el grupo de deterioro cognitivo moderado se encontraron correlaciones significativas negativas entre la conducta de prehensión y la frecuencia de trastornos del sueño y entre la conducta de prehensión y frecuencia de alucinaciones.

A menor puntaje en el ítem de conducta de prehensión (indicativo de patología), mayor la frecuencia de trastornos del sueño y alucinaciones. Se ha reportado en etapas más avanzadas de la enfermedad de Alzheimer la presencia de alucinaciones y de trastornos de sueño (Mesulam, 2000), que pueden deberse a una degeneración cortical difusa más avanzada, que afecta los lóbulos frontales. La presencia de la conducta de prehensión en pacientes con dicha degeneración sería un hallazgo probable.

Además, se encontró en este mismo grupo una correlación significativa positiva entre la fluencia verbal y la frecuencia de trastornos de alimentación. A mayor puntaje en el ítem de fluencia verbal (indicativo de preservación de la capacidad de generación de información), mayor frecuencia de trastornos de la alimentación, en el grupo que sufre de un deterioro cognitivo modera- 
do. Este hallazgo no fue esperado, ya que una de las funciones ejecutivas más afectadas es justamente la capacidad de generar palabras o flexibilidad mental, medida a través de la fluencia verbal, que se esperaría altamente deteriorada en este grupo de severidad del deterioro cognitivo.

Además, la aparición de cambios en el apetito se presenta en etapas más avanzadas de la enfermedad (Mesulam, 2000), etapa en la que justamente se observa también esta disminución de la fluencia verbal.

En el grupo deterioro cognitivo severo se encontró una correlación significativa perfecta entre el ítem Go-No go y la frecuencia de exaltación/euforia. A mayor puntaje en el ítem de GoNo go (indicativo de preservación de la capacidad de control de impulsos), mayor frecuencia de conductas de exaltación/euforia severa.

Este resultado no fue esperado, ya que, aunque se ha descrito la pérdida de la capacidad de controlar impulsos en etapas más avanzadas de la enfermedad de Alzheimer, este subgrupo presenta la preservación de esta capacidad.

Además, las conductas exaltadas son frecuentes en pacientes que sufren de una degeneración de los lóbulos frontales, que justamente afecta también la capacidad de controlar impulsos.

\section{CONCLUSIONES}

De acuerdo con lo expuesto, se podrían hacer las siguientes conclusiones:

En una muestra de pacientes con enfermedad de Alzheimer que alcanzan un deterioro cognitivo moderado, se observa una afectación severa de las funciones ejecutivas, alcanzando puntajes en la FAB equiparables con pacientes diagnosticados con demencia frontotemporal.

El desempeño de la muestra es indicativo de una disfunción ejecutiva desde etapas tempranas de la enfermedad, correspondientes a un deterioro cognitivo leve. Este hallazgo es consistente con varias investigaciones que sustentan la actual noción de una disfunción ejecutiva en la enfermedad de Alzheimer desde las etapas iniciales de la enfermedad.

La función ejecutiva que mostró desempeños alterados desde el subgrupo de deterioro cognitivo leve fue la capacidad de razonamiento abstracto, es decir, encontrar similaridades abstractas entre objetos presentados (Conceptualización), hallazgo que ha sido reportado en muestras de pacientes con Alzheimer en etapas tempranas (Baudic et al., 2006).

Se encuentra la presencia de síntomas neuropsiquiátricos desde etapas tempranas de la enfermedad, específicamente agitación, ansiedad, apatía e irritabilidad. 
Los síntomas neuropsiquiátricos de ansiedad, apatía, depresión, irritabilidad y agitación se muestran consistentemente a lo largo de varios estudios como característicos del perfil neuropsiquiátrico de la enfermedad de Alzheimer.

Existen correlaciones estadísticamente significativas entre la disfunción en funciones ejecutivas específicas y algunos síntomas neuropsiquiátricos. Muchas de estas correlaciones se sustentan en la descripción de la patología frontal, clásicamente estudiada en casos de lesión en las áreas frontales. Es decir, los trastornos del comportamiento y los disejecutivos observados en la presente investigación están bastante descritos en la literatura como parte del perfil característico de pacientes con lesiones frontales.

Las correlaciones significativas negativas encontradas entre síntomas neuropsiquiátricos y disfunción ejecutiva pueden explicarse como expresiones de una patología de los lóbulos frontales, en este caso, de etiología degenerativa, propia de la enfermedad de Alzheimer. Este hallazgo es consistente con otras investigaciones que han relacionado la aparición de síntomas neuropsiquiátricos y la disfunción ejecutiva.

Se hallaron correlaciones positivas no esperadas, que plantean interrogantes interesantes y son candidatas a estudios posteriores.
Las relaciones entre la disfunción ejecutiva y la frecuencia de síntomas neuropsiquiátricos se dan a lo largo de todos los subgrupos, sugiriendo una posible independencia de dicha relación con respecto al deterioro cognitivo global. Este hallazgo fue reportado por Chen et al. (1998).

\section{REFERENCIAS}

Aalten, P.; De Vugt, M.; Jaspers, N.; Jolles, J. \& Verhey, F. (2005). The course of neuropsychiatric symptoms in dementia. Part II: Relationships among behavioural sub-syndromes and the influence of clinical variables. International Journal of Geriatric Psychiatry; 20 (6), 531-536.

Alarcón, R. (1991). Métodos y diseños de investigación del comportamiento. Lima: Universidad Cayetano Heredia.

Alzheimer's Association (2005). <www.alz.org>.

American Health Assistance Foundation, 2005. 〈www.ahaf.org>.

Amieva, H.; Jacmin-Gadda, H.; Orgogozo, J; Le Carret, N.; Helmer, C.; Letenneur, L.; Barberger-Gateau, P.; Fabrigoule, L. \& Dartigues, J. (2005). The 9 year cognitive decline before dementia of the Alzheimer type: A prospective populationbased study. Brain, 128 (5), 10931101. 
Artaso-Irigoyen, B.; Goñi-Sarries, A. \& Gómez-Martínez, A. (2004). Síntomas neuropsiquiátricos en el síndrome demencial. Rev Neurol, 38 (6), 506-510.

Baudic S.; Barba Gd.; Thibaudet, Mc.; Smagghe, A.; Remy P. \& Traykov L. (2006). Executive function deficits in early Alzheimer's disease and their relations with episodic memory. Arch Clin Neuropsychol, 21 (1), 15-21.

Bell Mcginty, S.; Podell, K.; Franzen, M.; Baird, A. \& Williams, M. (2002). Standard measures of executive function in predicting instrumental activities of daily living in older adults. International Journal of Geriatric Psychiatry, 17 (9), 828-834.

Benoit, M.; Robert, P.; Staccini, P.; Brocker, P.; Lechowshi, L. \& Vellas, B. (2005). One year longitudinal evaluation of neurospychiatric symptoms in Alzheimer's disease. The real Fr. Study. The Journal of Nutrition, Health and Agin, 9 (2), 95-99.

Binetti, G.; Magni, E. \& Padovani, A. (1996). Executive dysfunction in early Alzheimer's Disease. $J$. Neurol Neurosurg Psychiatry, 60, 91-93.

Cannata, A.; Alberoni, M.; Franceschi, M. \& Mariani, C. (2002). Frontal impairment in subcortical ischemic vascular dementia in comparison to Alzheimer's disease. Dementia and geriatric cognitive disorders; 13 (2), 101-111.

Chen, St.; Sultzer, Dl. \& Hinkin, Ch. (1998). Executive dysfunction in Alzheimer's Disease: association with neuropsychiatric symptoms and functional impairment. $J$. Neuropsychiatry Clin Neurosci, 10, 426-432.

Collette, F. \& Van Der Linden, S. (1999). Executive Dysfunction in Alzheimer's Disease. Cortex, 35, 57-72.

Coolican, H. (1994). Métodos de investigación y estadística en psicología. México D.F.: El Manual Moderno.

Craig, D.; Mirakhur, A.; Hart, D.; McIlroy, S. \& Passmore, A. (2005) A cross-sectional study of neuropsychiatric symptoms in 435 patients with Alzheimer's disease. Am J Geriatr Psychiatry, 13 (6), 460-468.

Cummings, J. (1993). Frontal subcortical circuits and humans behavior. Arch Neuro, 50, 873-880.

Cummings, J. (2003). Toward a molecular neuropsychiatry of Neurodegenerative diseases. Ann Neurol, 54, 147-154.

Cummings, J. (2005). Mild cognitive impairment and neuropsychiatric symptoms. Simposio "Actualiza- 
ción en enfermedades neurodegenerativas: Demencias y enfermedades extrapiramidales". Santiago de Chile: Unidad de Neurología, Clínica Las Condes, abril de 2005.

Cummings, J.; Darkins, A.; Hill, M. \& Benson, D. (1988). Alzheimer's disease and Parkinson's disease: Comparison of speech and language alterations. Neurology, 38 (5), 680-684.

Cummings, J.; Dekosky, S. (1997). The Neuropsychiatric Inventory: Assessing psychopatolgy in dementia patients. Managing Alzheimer's disease. Neurology; 48 (5, SUP6), S10-S16.

Cummings, J.; Mega, M.; Gray, K.; Rosenberg Thompson, S.; Carusi, D. \& Gornbein, J. (1994). The Neuropsychiatric inventory: comprehensive assessment of psychopathology in dementia. Neurolog, 44 (12): 2308-2314.

Dalgleish, T. (2004). The emotional brain. Nature reviews, 5, 582-589.

Davis, K. Dementia. En: Chaney, D.; Nestler, E. \& Bunney, B. (1999). Neurobiology of mental illness. Nueva York: Oxford University Press.

De Toledo, M.; Bermejo-Pareja, F.; Vega-Quiroga, S. \& Muñoz-García, D. (2004). Alteraciones del comportamiento en la enfermedad de Al- zheimer. Rev Neurol, 38 (10), 901-905.

Demencia tipo Alzheimer. En: Biopsi-

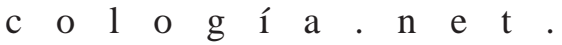
<http://www.biopsicologia.net/>.

Diccionario de psicología. En: $<$ http://www.psicoactiva.com/dicci o_b.htm>

Dubois, B.; Slachevsky, A.; Litvan, I. \& Pillon, B. (2000). The FAB: Frontal Assessment Battery at bedside. Neurology, 55 (11), 16211626.

Evolución histórica del concepto de demencia. En: Biopsicología.net $<$ http://www.biopsicologia.net/fich as/page_4747.html>.

Folstein, M.; Folstein, S. \& Mchugh, P. (1975). Mini Mental State: A practical method for grading the cognitive status of patients for the clinician. Journal of Psychiatric Research 12, 189-198.

Frances, A.; First, M. \& Pincus, H. (1997). Manual diagnóstico y estadístico de los trastornos mentales DSM-IV. Barcelona: Masson.

Frisoni, G.; Rozzini, L.; Gozetti, A.; Binetti, G.; Zanetti, O.; Bianchetti, A.; Trabucchi, M. \& Cummings, J. (1999). Behavioral syndromes in Alzheimer's disease: Description and correlates. Dementia and geriatric cognitive disorders, 10 (2), 130-138. 
Fuentes, P. (2005). Enfermedad de Alzheimer. Santiago de Chile: Unidad de Neurología Cognitiva y Demencias, Servicio de Neurología, Hospital del Salvador, 2005.

Fuentes, P. \& Slachevsky, A. (2005). Enfermedad de Alzheimer: Actualización en terapia farmacológica. Rev Méd Chile, 133, 224-230.

Gonzales, J. (2005). Psicobiología de la memoria. Santiago de Chile: Programa de Memoria, Universidad Católica de Chile.

Goodwin, C. J. (1998). Research in psychology: Methods and designs. Nueva York: John Wiley.

Hamilton, R. (2003). Las otras demencias: Neuropatología de las demencias distintas a la enfermedad de Alzheimer. Rev Neurol, 37 (2), 130-139.

Harwood, Dg.; Barker, Ww.; Ownby, Rl. \& Duara, R. (2000). Relationship of behavioral and psychiatric symptoms to cognitive impairment an functional status in Alzheimer's Disease. Int J Geriatr Psychiatry, 15, 393-400.

Harwood, G.; Sultzer, L. \& Wheatley, V. (2000). Impaired insight in Alzheimer's disease: Association with cognitive deficits, psychiatric symptoms and behavioral disturbances. Neuropsychiatry, neuropsychology and behavioral neurology, 13 (2), 83-88.
Hodges, J. (1994). Cognitive assessment for clinicians. Nueva York: Oxford University Press.

Hogan, D. \& Ebly, E. (1995). Primitive reflexes and dementia: Results from the Canadian Study of Health and Aging. Age Ageing, 24 (5), 375-81.

Holthoff, V.; Beuthien-Baumann, B.; Kalbe, E.; Ludecke, S.; Lenz, O.; Zundorf, G.; Spirling, S.; Schierz, K.; Winiecki, P. \& Herholz, K. (1969). Regional cerebral metabolism in early Alzheimer's disease with clinically significant apathy or depression. Biological psychiatry, 57 (4), 412-421.

Instituto Nacional de Estadísticas (INE). XVII Censo Nacional de Población y Vivienda 2002. Santiago de Chile.

Jódar-Vicente, M. (2004). Funciones cognitivas del lóbulo frontal. XXX Reunión de la Sociedad Española de Neurología Pediátrica. Rev Neurol, 39 (2), 178-182.

Johnson, J.; Head, E.; Kim, R.; Starr, A. \& Cotman, C. (1999). Clinical and pathological evidence for a frontal variant of Alzheimer disease. Arch Neurol, 56, 1233-1239.

Karlawish, J. \& Clark, C. (2003). Diagnostic evaluation of elderly patients with mild memory problems. Annals of Internal Medicine, 138, 411-419. 
Kaufer, D.; Cummings, J.; Ketchel, P.; Smith, V.; Macmillan, A.; Shelley, T.; López, O. \& Dekosky, S. (2000). Validation of the NPI-Q, a brief clinical form of the Neuropsychiatric Inventory. Journal of Neurology, Neurosurgery and Psychiatry, 69 (2), 167-171.

Kaufer, D. \& Dekoksky, S. (1999). Diagnostic classifications: relationships to the neurobiology of dementia. En: Charney, D.; Nestler, E. \& Bunney, B. Neurobiology of mental illness. Nueva York: Oxford University Press.

Kerlinger, F. (1991). Investigación del comportamiento. Técnicas y metodología. México: McGraw-Hill.

Lezak, M. (1995). Neuropsychological Assessment. Nueva York: Oxford University Press.

Lezak, M.; Howieson, D. \& Loring, D. (2004). Neuropsychological Assessment. Nueva York: Oxford University Press.

Lhermitte, F.; Pillon, B. \& Serdaru M. (1986). Human autonomy and the frontal lobes. Part I. Imitation and utilization behavior: A neuropsychological study of 75 patients. Ann Neurol, 19, 326-334.

Lipton, A.; Omán, K.; Womack, K.; Hynan, L.; Ninman, E. \& Lacritz, L. (2005). Subscores of the FAB differentiate frontotemporal lobar degeneration from AD., 65 (5), 726-731.

Maluenda, F. \& Riveros, R. (2005). Descripción de síntomas neuropsiquiátricos en pacientes con demencia vascular severa. Tesis de licenciatura en psicología. Santiago de Chile: Universidad de Chile.

Mapou, R. L. (1995). A cognitive framework for neuropsychological assessment. En: Mapou, R. L. \& Spector, J. Clinical neuropsychological assessment: A cognitive approach. Nueva York: Plenum Press.

McDowell, I. \& Newell, C. (1996). Measuring health. Nueva York: Oxford University Press.

McGuigan, F. J. (1996). Psicología experimental. Métodos de investigación. México: Prentice Hall.

McKhann, G.; Drachman, D.; Folstein, M.; Katzman, R.; Price, D. \& Stadlan, E. M. (1984). Clinical diagnosis of Alzheimer's disease: report of the NINCDS-ADRDA Work Group under the auspices of Department of Health and Human Services Task Force on Alzheimer's Disease. Neurology, 34: 939-944.

Mega, M.; Cummings, J.; Fiorello, T. \& Gornbein, J. (1996). The spectrum of behavioral changes in Alzheimer's disease. Neurology; 46 (1), 130-135. 
Mega, M. \& Cummings, J. (1994). Frontal subcortical circuits and neuropsychiatric disorders. J. Neuropsychiatry Clin Neurosci, 6 , 358-370.

Mesulam, M. (2000). Principles of behavioral and cognitive neurology. Nueva York: Oxford University Press.

Miller, B.; Chang, L.; Oropilla, G. \& Mena, I. (1994). Alzheimer's disease and frontal lobe dementias. En: Coffey, C. \& Cummings, J. Textbook of geriatric psychiatry. Washington DC: American Psychiatric Press.

Mirakhur, A.; Craig, D.; Hart, D.; Mcilroy, S. \& Passmore, A. (2004). Behavioral and psychological syndromes in Alzheimer's disease. International Journal of Geriatric Psychiatry, 19 (11), 1035-1039.

Morris, R. \& Worsley, C. (2003). Neuropsychological presentation of Alzheimer's disease and other neurodegenerative disorders. En: Halligan, P.; Kischka, U. \& Marshall, J. Handbook of Clinical Neuropsychology. Nueva York: Oxford University Press.

Nestor, P.; Scheltens, P. \& Hodges, J. (2001). Advances in the early detection of Alzheimer's disease. Nature Reviews in Neurocience, 5, 534-541.
Neuropatología de las demencias. Revista de Neuropsiquiatría (2005). <http://www.scielo.org.pe>.

Olarzarán Rodríguez, J. La evaluación neuroconductual. España. <http:// www.neurologia.rediris.es/con$\mathrm{g} \mathrm{r}$ e s o - / c o n f e r e n cias/neuropsicologia.html>.

Organización Panamericana de la Salud (2003). Guía clínica para atención primaria a las personas mayores. Washington DC.

Paulsen, J.; Ready, R. \& Stout, J. (2000). Neurobehaviors and psychotic symptoms in Alzheimer's disease. J Int Neuropsychol Soc., 6, 815-820.

Piccininni, M.; Di Carlo, A.; Baldereschi, M.; Zaccara, G. \& Inzitari, D. (2005). Behavioral and psychological symptoms in Alzheimer's disease: Frequency and relationship with duration and severity of the disease. Dementia and geriatric cognitive disorders, 19 (5-6), 276-281.

Pineda, D. La función ejecutiva y sus trastornos. España. $<$ http://neurologia.rediris.es/congre $\mathrm{s}$ o - $1 /$ c o n f e r e n cias/neuropsicologia.html>.

Pineda, D. (2000). La función ejecutiva y sus trastornos. I Congreso Virtual Iberoamericano de neurología. Rev Neurol, 30 (8), 764-768.

Porter, V.; Buxton, W.; Fairbanks, L.; 
Strickland, T.; O' Connor, S.; Rosenberg Thompson, S. \& Cummings, J. (2003). Frequency and characteristics of anxiety among patients with Alzheimer's disease and related dementias. The Journal of Neuropsychiatry and Clinical Neurosciences; 15 (2): 180-186.

Price, D.; Markowska, A.; Koliatsos, V.; Frick, K.; Borchelt, D.; Martin, L.; Kawas, C. \& Sisodia, S. (1999). Alzheimer's disease and animal models. En: Charney, D.; Nestler, E. $\&$ Bunney, B. Neurobiology of mental illness. Nueva York: Oxford University Press.

Quiroga, P.; Albala, C. \& Klaansen, G. (2004). Validation of a screening test for age associated cognitive impairment in Chile. Revista Médica de Chile, 132 (4), 467-478.

Rajah, M. \& D'Esposito, M. (2005). Region-specific changes in pre frontal cortex function with age: A review of PET and MRI studies on working and episodic memory. Brain 128, 1964-1983.

Reichman, W. (1994). Nondegenerative dementing disorders. En: Coffey, C. \& Cummings, J. Textbook of geriatric psychiatry. Washington DC: American Psychiatric Press.

Robert, P.; Clairet, S.; Benoit, M.; Koutaich, J.; Bertogliati, C.; Tible, O.; Caci, H.; Borg, M.; Brocker, P.
\& Bedoucha, P. (2002). Apathy inventory: Assessment of apathy and awareness in Alzheimer's disease, Parkinson's disease and mild cognitive impairment. International Journal of Geriatric Psychiatry; 17, 1099-1105.

Rodríguez-Del Álamo, A.; Catalan, M. \& Carrasco, L. (2003). FAB: Aplicación preliminar española de la batería neuropsicológica de evaluación de funciones frontales a 11 grupos de pacientes. Rev Neurol, 36 (7), 605-608.

Rosenberg, P.; Onyike, C.; Katz, I.; Porsteinsson, A.; Mintzer, J.; Schneider, L.; Rabins, P.; Meinert, C.; Martin, B. \& Lyketsos, C. (2005). Clinical application of operationalized criteria for "Depression of Alzheimer disease". International Journal of Geriatric Psychiatry, 20 (2), 119-127.

Sánchez, H. \& Reyes, C. (1998). Metodología y diseños de investigación científica. Lima: Editorial Mantaro, 1998.

Senanarong, V.; Cummings, J.; Fairbanks, L.; Mega, M.; Masterman, D.; O' Connor, S. \& Strickland, T. (2004). Agitation in Alzheimer's disease is a manifestation of frontal lobe dysfunction. Dementia and Geriatric Cognitive Disorders, 17 (1-2), 14-20.

Senanarong, V.; Poungvarin, N.; 
Jamjumras, P.; Sriboonroung, A.; Danchaivijit, C.; Udomphanthuruk, S. \& Cummings, J. (2005). Neuropsychiatric symptoms, functional impairment and executive ability in Thai patients with Alzheimer's disease. International psychogeriatrics, 17 (1), 81-90.

Sevush, S.; Peruyera, G.; Bertran, A. \& Cisneros, W. (2003). A three factor model of cognition in Alzheimer's disease. Cognitive and Behavioral Neurology, 16 (2), 110-117.

Sgaramella, T.; Borgo, F. \& Mondini, S. (2001). Executive déficits appearing in the initial state of Alzheimer's Disease. Brain Cog.; 46, 264-268.

Sherrington, R.; Hyslop, P.; Hutton, M.; Perez-Tur, J. \& Hardy, J. The molecular biology of Alzheimer's disease. (1999). En: Charney, D.; Nestler, E. \& Bunney, B. Neurobiology of mental illness. Nueva York: Oxford University Press.

Sierra Bravo, R. (1995). Técnicas de investigación social, teoría y ejercicios. Madrid: Paraninfo.

Slachevsky, A. \& Alegría, P. (2005). Síndromes lobares. Diplomado de Neuropsicología Versión II. Santiago de Chile: Universidad Católica de Chile.

Slachevsky, A.; Pérez, C.; Silva, J.; Orellana, G.; Prenafeta, M.; Alegría, P. \& Peña, M. (2005). Córtex pre- frontal y trastornos del comportamiento: Modelos explicativos y métodos de evaluación. Rev Chil Neuro-Psiquiat, 43 (2), 109-121.

Slachevsky, A.; Villalpando, J.; Saranzin, M.; Hanh-Barma, V.; Pillon, B. \& Dubois, B. (2004). Frontal Assessment Battery and differential diagnosis of Frontotemporal Dementia and Alzheimers Disease. Arch Neurol, 61, 1104-1107.

Spreen, O. \& Strauss, E. (1998). A compendium of neuropsychological tests. Second edition. Administration, norms and commentary. Nueva York: Oxford University Press.

Starkstein, S.; Garau, M. \& Cao, A. (2004). Prevalence and clinical correlates of disinhibition in dementia. Cognitive and Behavioural Neurology, 17 (3), 139-147.

Sultzer, D.; Brown, C.; Mandelkern, M.; Mahler, M.; Mendez, M.; Chen, S. \& Cummings, J. (2003). Delusional thoughts and regional Frontal/Temporal Cortex Metabolism in Alzheimer's disease. American Journal of Psychiatry, 160, 2.

Swanberg, M.; Tractenberg, R.; Mohs, R.; Thal, L. \& Cummings, J. (2004). Executive dysfunction in Alzheimer's Disease. Arch Neurol, 61, 556-560.

Takechi, H.; Yamada, H.; Sugihara, Y. \& Kita, T. (2006). Behavioral and psychological symptoms, cognitive 
impairment and caregiver burden related to Alzheimer's disease patients treated in an outpatient memory clinic. Nippon Ronen Igakkai Zasshi, 43 (2), 207-16.

Tekin, S.; Mega, M. \& Masterman, D. (2001). Orbitofrontal and anterior cingulate cortex neurofibrillary tangle burden is associated with agitation in Alzheimer disease, 49 (3), 355-61.

Van Reekum, R.; Stuss, D. \& Ostrander, L. (2005). Apathy; Why care? The Journal of Neuropsychiatry and Clinical Neuroscience, 17 (1), 7-19.

Velázquez Quintana, M. (2001). Guía para elaborar un proyecto reinvestigación. Parte II. Revista Mexicana de Neurociencia, 2 (1), 51-53.

Waltz, J.; Knowlton, B; Holyoak, K.; Boone, K.; Back Madruga, C.; Mcpherson, S.; Mastermann, D.; Chow, T.; Cummings, J. \& Miller,
B. (2004). Relational integration and executive function in Alzheimer's disease. Neuropsychology, 18 (2), 296-305.

Wells, C. \& Whitehouse, P. (1996). Cortical Dementia. En: Fogel; Schiffer, R. \& Rao, S. Neuropsychiatry. Baltimore: Williams \& Wilkins, Baltimore.

Wenk, G. (1999). Functional neuroanatomy of learning and memory. En: Charney, D.; Nestler, E. \& Bunney, B. Neurobiology of mental illness. Nueva York: Oxford University Press.

Wilson, RS.; Gilley, D.W. \& Bennett, DA. (2000). Hallucinations, delusions and cognitive decline in Alzheimer's Disease. J Neurol Neurosurg Psychiatry, 69, 172-177.

World Health Organization. International statistical classification of diseases and related health problems. Décima versión revisada para el 2006. <http://www.who. int/classifications/apps/icd/icd10on 


\section{ANEXOS}

\section{MINIMENTAL TEST DE FOLSTEIN}

"A continuación le haré algunas preguntas para apreciar cómo funciona su memoria. Algunas son muy simples y otras un poco más complejas. Le pido que trate de responder lo mejor que pueda". (Otorgar 10 segundos por cada respuesta) (Contar 1 punto por respuesta correcta)

\begin{tabular}{|l|l|l|l|l|l|l|}
\hline & Sí & No & N/S & Respuesta & Nota 0/1 \\
\hline 1 & ¿En qué año estamos? & & & & & \\
\hline 2 & ¿En qué estación del año estamos? & & & & & \\
\hline 3 & ¿En qué mes estamos? & & & & \\
\hline 4 & ¿Cuál es la fecha de hoy? & & & & & \\
\hline 5 & ¿Qué día de la semana es hoy? & & & & \\
\hline 6 & ¿En qué comuna estamos? & & & & \\
\hline 7 & ¿A qué ciudad pertenece esta comuna? & & & & \\
\hline 8 & ¿En qué provincia o región está situada esta comuna? & & \\
\hline 9 & $\begin{array}{l}\text { ¿Cuál es el nombre del hospital (o clínica) en donde esta- } \\
\text { mos?, o ¿en qué lugar físico estamos? }\end{array}$ & & & & \\
\hline 10 & ¿En qué piso estamos? & & & & \\
\hline \multicolumn{2}{|l|}{} & & & \\
\hline
\end{tabular}

"Le voy a decir 3 palabras; usted las repetirá y tratará de retenerlas en su mente ya que en un rato más le pediré que me las vuelva a repetir".

(Dar las palabras con 1 segundo de intervalo, pronunciándolas en voz alta y articulando bien): "Cigarro, Flor, Puerta. Repita las tres palabras".

\begin{tabular}{|c|l|c|c|c|c|c|}
\hline & & Sí & No & N/S & Respuesta & Nota 0/1 \\
\hline 11 & Cigarro & & & & & \\
\hline 12 & Flor & & & & & \\
\hline 13 & Puerta & & & & Total & $/ 3$ \\
\hline \multicolumn{7}{|l|}{} \\
\hline
\end{tabular}

(Contar 1 punto por cada palabra correctamente repetida en el primer ensayo.) (Otorgar 20 segundos para la respuesta. Si el sujeto no repite las 3 palabras al primer ensayo, dárselas nuevamente, hasta que las 3 palabras sean aprendidas. Máximo 5 ensayos.)

\begin{tabular}{|l|l|}
\hline Número de respuestas correctas & \\
\hline Número de repeticiones & \\
\hline
\end{tabular}


"Ahora le pido que cuente hacia atrás partiendo de 100 y restando cada vez 7". (En esta prueba hay que darle al paciente las máximas facilidades; está permitido simplificar la consigna: ¿cuánto es 100-7? También está permitido recordar cada resta si el paciente pierde la consigna: ¿y 93-7, cuánto es? Si el paciente da una respuesta errónea preguntarle: ¿Está seguro del resultado?, y permitir la corrección: la prueba debe detenerse después de dos errores.) (Otorgar 10 segundos por cada respuesta.)

\begin{tabular}{|c|l|l|l|l|l|c|}
\hline & & Sí & No & N/S & Respuesta & Nota 0/1 \\
\hline 14 & 93 & & & & & \\
\hline 15 & 86 & & & & & \\
\hline 16 & 79 & & & & & \\
\hline 17 & 72 & & & & & \\
\hline 18 & 65 & & & & & Total \\
\hline \multicolumn{6}{|l|}{} \\
\hline
\end{tabular}

Contar 1 punto por cada resta exacta $(93,86,79,72,65)$.

Si el paciente no obtiene el máximo de puntos pedirle: (Distracción) "Dígame al revés la palabra CIELO comenzando por la última letra".

(Anotar el número de letras dadas en el orden correcto: este resultado no figura en el resultado global.)

\begin{tabular}{|l|l|l|l|l|l|l|}
\hline & & Sí & No & N/S & Respuesta & Nota 0/5 \\
\hline $18 \mathrm{a}$ & Cielo & & & & & \\
\hline
\end{tabular}

"Dígame por favor cuáles eran las tres palabras que repitió hace un rato" (Otorgar 10 segundos para responder. Contar 1 punto por cada respuesta correcta.)

\begin{tabular}{|c|l|l|l|l|l|c|}
\hline & & Sí & No & N/S & Respuesta & Nota 0/3 \\
\hline 19 & Cigarro & & & & & \\
\hline 20 & Flor & & & & & \\
\hline 21 & Puerta & & & & Total & 13 \\
\hline \multicolumn{6}{|r|}{} \\
\hline
\end{tabular}

Si el paciente no recuerda las palabras, se le presentan dos palabras para estudiar el reconocimiento: "La palabra que le había dicho era": (No se dan puntos en caso de respuestas correctas.)

\begin{tabular}{|l|l|l|l|l|l|}
\hline & & Sí & No & N/S & Respuesta \\
\hline & Cigarro o llave & & & & \\
\hline & Mesa o flor & & & & \\
\hline & Puerta o pelota & & & & \\
\hline
\end{tabular}

(Mostrar 1 lápiz.) (Dar 1 punto en caso de respuesta correcta.)

\begin{tabular}{|l|l|l|l|l|l|l|l|}
\hline & & Sí & No & N/S & Respuesta & Nota 0/1 \\
\hline 22 & ¿Cúal es el nombre de este objeto? & & & & & \\
\hline
\end{tabular}


(Mostrar 1 reloj.) (Dar 1 punto en caso de respuesta correcta.)

\begin{tabular}{|l|l|l|l|l|l|l|}
\hline & & Sí & No & N/S & Respuesta & Nota 0/1 \\
\hline 23 & ¿Cúal es el nombre de este objeto? & & & & & \\
\hline
\end{tabular}

(Mostrar 1 reloj.) (Dar 1 punto en caso de respuesta correcta.)

\begin{tabular}{|l|l|l|l|l|l|l|}
\hline & & Sí & No & N/S & Respuesta & Nota 0/1 \\
\hline 24 & "No hay ni si, ni no, ni pero" & & & & & \\
\hline
\end{tabular}

(Contar punto si la repetición es totalmente correcta. Contar 0 al menor error)

Pasar al paciente una hoja de papel (ver anexo) en la que está escrito en letras grandes y marcadas: CIERRE LOS OJOS. Se da la consigna siguiente: "Lea este papel y haga lo que está marcado" (Otorgar 10 segundos para la respuesta.) (Contar 1 punto solamente si el sujeto cierra los ojos.)

\begin{tabular}{|l|l|l|l|l|l|l|}
\hline & Sí & No & N/S & Respuesta & Nota 0/1 \\
\hline 25 & Cierre los ojos & & & & & \\
\hline
\end{tabular}

Pase al paciente una hoja de papel diciéndole:

\begin{tabular}{|l|l|l|l|l|l|l|}
\hline 26 & Sí & No & N/S & Respuesta & Nota 0-3 \\
\hline $\begin{array}{l}\text { "Tome este papel con la mano derecha, dó- } \\
\text { blelo dos veces usando las dos manos, } y \\
\text { luego tírelo al suelo" }\end{array}$ & & & & & & \\
\hline
\end{tabular}

(Aceptar 1 punto por ítem correctamente ejecutado: máximo 3 puntos.) (Si el paciente se detiene y pregunta lo que debe hacer, responder: "lo que yo le he pedido hacer".)

Pase al paciente una hoja de papel en blanco y un lápiz. Mostrarle el dibujo (ver hoja anexa) y pedirle:

\begin{tabular}{|l|l|l|l|l|l|l|}
\hline & & Sí & No & N/S & Respuesta & Nota 0/1 \\
\hline 27 & "Por favor copie este dibujo" & & & & & \\
\hline
\end{tabular}

(Máximo de tiempo 60 segundos) (Contar 1 punto solamente si la copia tiene todos los ángulos de cada figura y si las figuras se cortan en dos lados diferentes)

Sobre la misma hoja de papel (ver hoja anexa) solicitarle al paciente:

\begin{tabular}{|l|l|l|l|l|l|l|}
\hline 28 & Sí & No & N/S & Respuesta & Nota 0/1 \\
pero una oración completa"
\end{tabular}

(Contar 1 punto si la frase tiene un sujeto, un verbo y un predicado. No se tomarán en cuenta las faltas de ortografía ni de sintaxis.) (Si el sujeto no escribe una frase completa, preguntarle: ¿esta es una frase?, y permitirle su corrección si está consciente de su error, siempre dentro del tiempo impartido. Máximo de tiempo: 60 segundos). 
CIERRE LOS OJOS

Anexo de pregunta 25

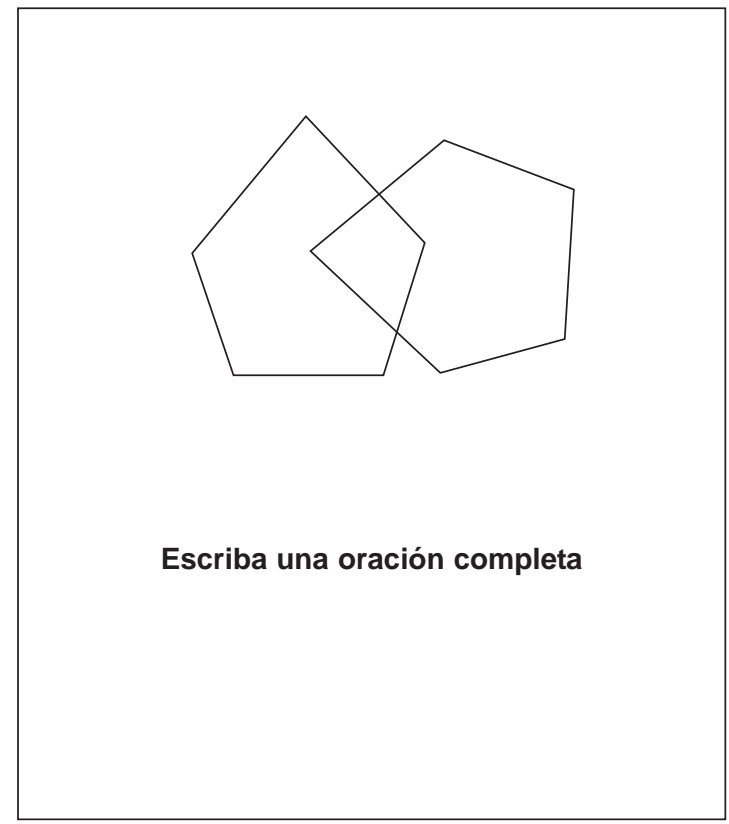

Anexo de preguntas 27 y 28 


\section{Frontal Assessment Battery FAB}

1. Semejanzas (conceptualización)

“¿En qué se parecen...?”

a. Un plátano y una naranja.

b. Una mesa y una silla.

c. Un tulipán, una rosa y una margarita.

Ayudar al paciente en caso de fracaso total: "no se parecen" o parcial: "los 2 tienen cáscara" en el primer ítem, no en los siguientes. Solo las respuestas de categoría (frutas, muebles, flores) se consideran correctas.

Puntaje: 3 correctas $=3 ; 2$ correctas $=2 ; 1$ correcta $=1 ;$ ninguna correcta $=0$

2. Fluidez léxica (flexibilidad mental)

"Diga todas las palabras que pueda (por ejemplo, animales, plantas y objetos, pero no nombres propios ni apellidos) que comiencen con A". Si no responde en los primeros 5 segundos decirle "por ejemplo, árbol". Si se detiene por más de 10 segundos, insista "cualquier palabra que empiece con A". Tiempo: 60 segundos. Las repeticiones, derivaciones (árbol, arbolito), nombres propios y apellidos no se cuentan.

Puntaje: 10 o más palabras $=3 ; 6$ a $9=2 ; 3$ a $5=1$; menos de $3=0$

3. Secuencias motoras (programación)

"Mire con atención lo que hago". El examinador frente al paciente realiza 3 veces la prueba de Luria (golpear con nudillo, canto y palma) con su mano izquierda. "Con su mano derecha haga lo mismo que yo, primero juntos, después solo". El examinador hace la serie 3 veces con el paciente y le dice "ahora haga lo mismo Ud. Solo".

Puntaje: 6 series consecutivas correctas $=3$; 3 a 5 series correctas $=2$; no lo hace solo, pero sí 3 series consecutivas con el examinador $=1$; no logra ni siquiera imitar 3 veces $=0$

4. Instrucciones conflictivas (sensibilidad a la interferencia)

"Cuando yo golpeo 1 vez, debe golpear 2 veces"; para asegurar que comprendió las instrucciones, se hace una serie de 3 ensayos: 1-1-1. "Cuando yo golpeo 2 veces, debe golpear una"; para asegurar que comprendió las instrucciones, se hace una serie de 2-2-2. El examinador realiza la siguiente serie: $1-1-2-1-2-2-2-1-1-2$.

Puntaje: $\sin$ errores $=3 ; 1$ o 2 errores $=2$; más de 2 errores $=1$;

si golpea igual que el examinadoral menos 4 veces consecutivas $=0$.

5. Go-No go (control inhibitorio)

"Cuando yo golpeo 1 vez, debe golpear 1 vez"; para asegurar que comprendió la instrucción, se hace una serie de 3 ensayos: 1-1-1. "Cuando yo golpeo 2 veces, no debe golpear"; para asegurar que comprendió la instrucción, se hace una serie de 3 ensayos: 2-2-2. El examinador realiza la siguiente serie: $1-1-2-1-2-2-2-1-1-2$.

Puntaje: $\sin$ errores $=3 ; 1$ o 2 errores $=2$; más de 2 errores $=1$;

golpea igual que el examinadoral menos 4 veces seguidas $=0$.

6. Conducta de prehensión (autonomía del ambiente)

El examinador se sienta frente al paciente, que tiene las manos sobre sus rodillas, con las palmas hacia arriba. El examinador acerca lentamente sus manos hasta tocar las del paciente para ver si se las toma espontáneamente. Si lo hace, dice "ahora, no me tome las manos" y vuelve a tocárselas. Puntaje: no le toma las manos $=3$; duda o pregunta qué tiene que hacer $=2$; las toma $\sin$ vacilar $=1$; llas toma aún después de decirle que no lo haga $=0$. 


\section{SíNTOMAS NEUROPSIQUIÁTRICOS (NPI-Q)}

Responda el siguiente cuestionario basado en cambios ocurridos desde que él/ella empezó a experimentar problemas de memoria:

Marque "No" si los síntomas no ocurren en la actualidad. Marque "Sí" solo si los síntomas ocurren en la actualidad.

Para cada ítem marcado con "Sí":

a) Relación de GRAVEDAD con el síntoma o síntomas (cómo afecta al paciente):

1: Leve (cambio evidente, pero no es significativo, fácil manejo de la situación).

2: Moderada (cambio significativo, pero no drástico, se hace más difícil controlar la situación).

3: Grave (cambio drástico, muy marcado, no se puede manejar la situación)...

b) Relación de su ESTRÉS con el síntoma/s (cómo le afectan emocionalmente a usted los síntomas):

0 : No existe afectación.

1: Mínima (poca afectación, no me representa un problema).

2: Leve (sin demasiada afectación, me las arreglo fácilmente).

3: Moderada (bastante afectación, no siempre puedo arreglármelas).

4: Grave (mucha afectación, dificultades para arreglármelas).

5: Muy grave (afectación extrema, el problema me vence).

DELIRIOS

¿Cree el paciente en cosas que no son ciertas, como por ejemplo que otras personas quieren robarle o quieren hacerle daño? ¿Dice que miembros de su familia no son quienes dicen ser o que su casa no es su casa?

No

GRAVEDAD: 123

EstRÉS: $\begin{array}{llllll}0 & 1 & 2 & 3 & 4 & 5\end{array}$

Alucinaciones

¿El paciente ve cosas o personas inexistentes o dice que escucha voces o ruidos inexistentes? ¿Habla con personas que no están realmente presentes?
Sí
No $\square$
Gravedad: 123
Estrés: $0 \begin{array}{lllll} & 1 & 2 & 3 & 4\end{array}$

Agitación / Agresión

¿El paciente insulta o se molesta con su cuidador con facilidad? ¿Se niega a cooperar o recibir ayuda en actividades, como por ejemplo bañarse o vestirse?
Sí
No $\square$
GRAVEDAD: 123
ESTRÉS: $\begin{array}{llllll}0 & 1 & 2 & 3 & 4 & 5\end{array}$

DEPRESIÓN / DISFORIA

¿El paciente está triste o bajo de moral? ¿Llora?

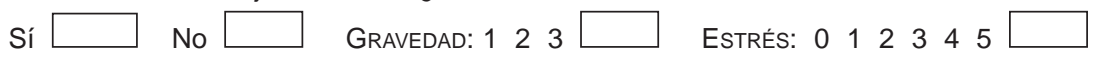

ANSIEDAD

¿El paciente está nervioso, inquieto, no puede relajarse, o está excesivamente tenso? ¿Dice que tiene como un nudo en el estómago o se inquieta cuando se separa de usted?

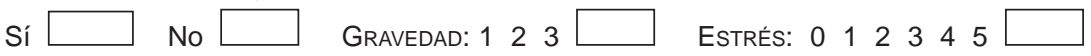

EXALTACIÓN / EUFORIA

¿Parece el paciente estar demasiado alegre? Se refiere a una alegría anormal, excesiva, diferente a como ha sido siempre

Sí

No

Gravedad: 123

Estrés: $0 \begin{array}{lllll}0 & 1 & 2 & 3 & 4\end{array}$ 
APATÍA / INDIFERENCIA

¿El paciente parece poco interesado, poco motivado para hacer cosas, menos activado que de costumbre, incluso habla menos?

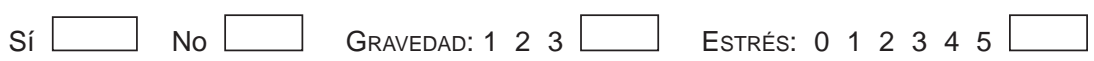

DESINHIBICIÓN

¿El paciente actúa impulsivamente, dice cosas que normalmente no se dicen o se hacen en público? (cosas que incluso pueden hacerle sentir "vergüenza").

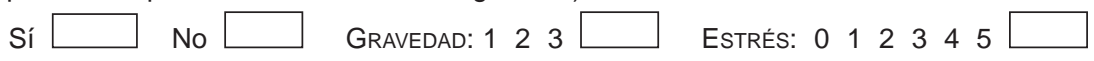

Irritabilidad /Labilidad

¿Está irritable o se molesta con facilidad? ¿Tiene "arranques" repentinos de mal humor o ira que no corresponden a su carácter habitual? ¿Se muestra impaciente?
Sí
No
GRAVEDAD: 123
ESTRÉS: $0 \begin{array}{llllll}0 & 1 & 2 & 3 & 4 & 5\end{array}$

\section{CONDUCTA MOTORA ANÓMALA}

¿El paciente se dedica a repetir actos como dar vueltas por la casa, abrir cajones o armarios o hacer otras cosas repetitivamente con la ropa, con los dedos o con otros objetos?

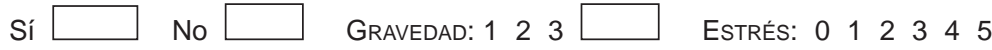

\section{SUEÑO}

¿Tiene dificultad para conciliar el sueño, se despierta durante la noche (no tener en cuenta si se levanta para ir al baño y vuelve a dormir), se levanta demasiado temprano? ¿Esto le lleva a dormir excesivamente durante el día?
Sí
No $\square$
GRAVEDAD: 123
Estrés: $0 \begin{array}{llllll}0 & 1 & 2 & 3 & 4 & 5\end{array}$

\section{ApETIto/Alimentación}

¿El paciente ha perdido o ganado apetito y/o peso, o ha cambiado de gustos en las comidas?

Si<smiles>C1CCC1</smiles>

No

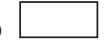

Gravedad: 123
ESTRÉS: $\begin{array}{lllllll}0 & 1 & 2 & 3 & 4 & 5\end{array}$ 